University of Warwick institutional repository: http://go.warwick.ac.uk/wrap This paper is made available online in accordance with publisher policies. Please scroll down to view the document itself. Please refer to the repository record for this item and our policy information available from the repository home page for further information.

To see the final version of this paper please visit the publisher's website. Access to the published version may require a subscription.

Author(s): CHRISTIAN BONATTI and EMMANUEL DUFRAINE Article Title: Équivalence topologique de connexions de selles en dimension 3

Year of publication: 2003

Link to published version: http://dx.doi.org/10.1017/S0143385703000130

Publisher statement: None 


\title{
Équivalence topologique de connexions de selles en dimension 3
}

\author{
CHRISTIAN BONATTI $\dagger$ et EMMANUEL DUFRAINE $\ddagger$ \\ $\dagger$ Laboratoire de Topologie, UMR 5584 du CNRS, B.P. 47 870, \\ 21078 Dijon Cedex, France \\ (e-mail:bonatti@u-bourgogne.fr) \\ $\$$ Mathematics Institute, University of Warwick, CV4 7AL Coventry, UK \\ (e-mail:dufraine@maths.warwick.ac.uk)
}

(Received 13 November 2002 and accepted in final form 17 March 2003)

Résumé. Nous donnons des invariants complets pour l'équivalence topologique de champs de vecteurs, en dimension 3, au voisinage d'une connexion (par des variétés invariantes de dimension 1) entre des selles possédant des valeurs propres complexes.

Abstract. We give a complete set of invariants for the topological equivalence of vector fields on 3-manifolds in the neighborhood of a connection by one-dimensional separatrices between two hyperbolic saddles having complex eigenvalues.

More precisely, let $X$ be a $C^{2}$ vector field on a 3-manifold, having two hyperbolic zeros $p, q$ of saddle type, such that $p$ admits a contracting complex eigenvalue $-c_{p}(1+i \alpha)$, $c_{p}>0, \alpha \neq 0$, and $q$ admits an expanding complex eigenvalue $c_{q}(1+i \beta), c_{q}>0$, $\beta \neq 0$. We assume that a one-dimensional unstable separatrix of $p$ coincides with a onedimensional stable separatrix of $q$, and we call the connection the compact segment $\gamma$ consisting of $p, q$ and their common separatrix (see Figure 1). Such a connection is a codimension two phenomenon.

The behaviour of a vector field $X$ in the neighborhood of the connection is given, up to topological conjugacy, by the linear part of $X$ in the neighborhood of $p$ and $q$ and by the transition map between two discs transversal to $X$ in those neighborhoods. First, we choose coordinates in the neighborhoods of $p$ and $q$ in order to put $X$ in canonical form and we show that, up to topological equivalence, we can assume that the transition map is linear. Then our main result is as follows :

- When the linear transition map (expressed in the chosen coordinates) is conformal or when its modulus of conformality is small (i.e. less than some function $\psi(\alpha, \beta)$ ), there is no topological invariant : every two such vector fields are topologically equivalent.

- $\quad$ On the other hand, when the modulus of conformality is greater than $\psi(\alpha, \beta)$, there are two topological invariants : one is equal to the ratio $\alpha / \beta$, the other one is related to the modulus of conformality of the transition map. 


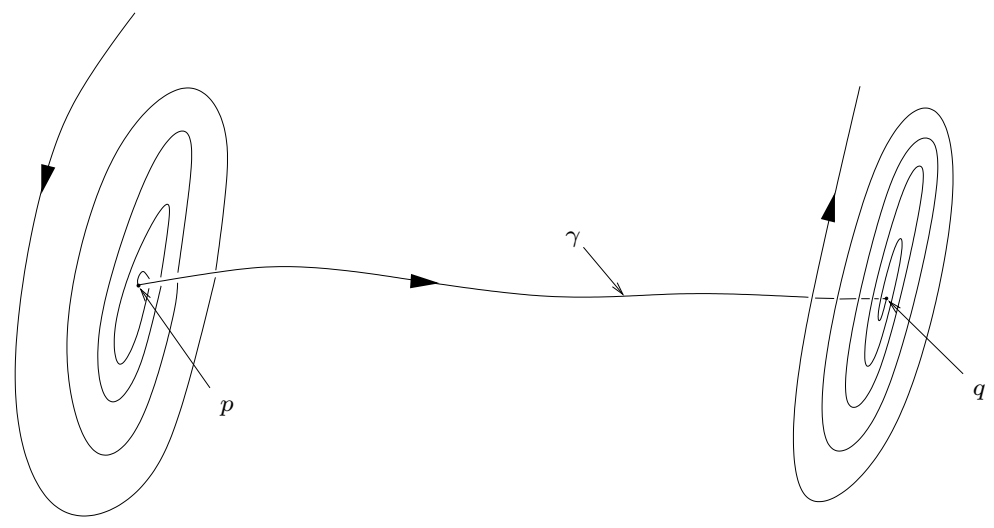

FIGURE 1. Portrait de phase au voisinage de la connexion.

\section{Introduction}

On considère des champs de vecteurs de classe $C^{2}$ sur des variétés de dimension 3 possédant une connexion de selles par des variétés invariantes de dimension 1 , et on cherche à donner une classification, modulo équivalence topologique, des champs de vecteurs au voisinage d'une telle connexion. Rappelons qu'en dimension 2, Palis (voir [7]) a exhibé une fonction des valeurs propres des selles apparaissant dans la connexion, qui donnait un invariant complet de conjugaison au voisinage de cette connexion. Sur une surface, il n'y a aucun invariant d'équivalence topologique : deux champs de vecteurs de classe $C^{1}$ sur une surface sont toujours topologiquement équivalents au voisinage d'une connexion de selles. De nombreux auteurs (voir par exemple [2, 8-10]) ont donné des invariants d'équivalence topologique de champs de vecteurs au voisinage d'une connexion de codimension 1, en toutes dimensions.

En dimension 3 , le phénomène que nous étudions est un phénomène de codimension 2 où la situation paraît extrêmement simple : on considère un champ de vecteurs $X$ de $\mathbb{R}^{3}$ possédant deux zéros hyperboliques $p$ et $q$ de type selle, tels que la variété instable de $p$ et la variété stable de $q$ soient de dimension 1 . Chacune de ces variétés invariantes de dimension 1 est constituée du zéro $p$ ou $q$ et de deux orbites régulières, appelées séparatrices. Notre champ $X$ possède une connexion entre les deux selles $p$ et $q$ : l'une des séparatrices instables de $p$ coïncide avec l'une des séparatrices stables de $q$. La connexion $\gamma$ entre les selles $p$ et $q$ est le segment compact formé des deux selles $p$ et $q$ et d'une séparatrice commune à $p$ et $q$.

Définition 1.1. On dit que deux champs de vecteurs $X$ et $\tilde{X}$ sont topologiquement équivalents au voisinage de connexions de selles $\gamma$ et $\tilde{\gamma}$, s'il existe un homéomorphisme $h$ d'un voisinage de $\gamma$ sur un voisinage de $\tilde{\gamma}$ tel que $h(\gamma)=\tilde{\gamma}$ et que l'image d'un segment d'orbite de $X$ orienté par $X$ est un segment d'orbite de $\tilde{X}$ positivement orienté.

On dira qu'ils sont positivement topologiquement équivalents si l'homéomorphisme $h$ peut être choisi préservant l'orientation. 
Le comportement (à équivalence topologique près) d'un champ au voisinage d'une connexion est essentiellement décrit par sa partie linéaire au voisinage des deux points selles, et par une transition $\mathcal{T}$, qui est l'holonomie du champ le long de la connexion entre deux disques transverses au champ et contenus dans des ouverts de linéarisation au voisinage des points singuliers.

Dans le cas où les valeurs propres associées aux points $p$ et $q$ sont toutes réelles, Vegter (voir $[\mathbf{1 1}, \mathbf{1 2}]$ ) montre que, génériquement, il n'y a pas de module de stabilité : toute perturbation du champ $X$, suffisamment $C^{1}$-petite et préservant la connexion, est topologiquement équivalente à $X$ au voisinage de la connexion. Ce résultat a été généralisé en toutes dimensions par Dias Carneiro et Palis (voir [4]).

Nous considérons ici le cas où les points selles $p$ et $q$ possèdent chacun une valeur propre complexe (non-réelle), stable pour $p$ (donc de partie réelle négative) et instable pour $q$ (de partie réelle positive). Nous donnons dans ce cadre une classification complète (modulo équivalence topologique), sans hypothèse de généricité. Pour cela nous montrons d'abord (voir la Proposition 2.1) que, dans ce cas, on peut supposer que la transition $\mathcal{T}$ est linéaire. On obtient alors un résultat assez surprenant :

- Quand la transition est une application conforme, ou que son défaut de conformité est petit, alors il n'y a aucun invariant : tous les champs sont topologiquement équivalents au voisinage de la connexion.

- Par contre, quand le défaut de conformité devient plus grand, alors apparaissent deux invariants : l'un est l'équivalent de l'invariant de Palis (en dimension 2) cité ci-dessus, et l'autre est directement lié au défaut de conformité de la transition.

D'autres motivations seront présentées dans la conclusion de cet article (voir les §§A.1 et A.2).

Afin de présenter notre résultat, nous devons donner une description précise du champ $X$ au voisinage de la connexion $\gamma$.

1.1. Choix de coordonnées au voisinage de la connexion et notations. D'après [1] ], pour tout point selle $p$ d'un champ de vecteurs de classe $C^{2}$ en dimension 3 , et possèdant une valeur propre complexe (non-réelle), il existe des coordonnées de classe $C^{1}$ sur un voisinage $U_{p}$ de $p$, dans lesquelles le champ s'écrit comme un champ de vecteurs linéaire.

Quitte à composer ce changement de coordonnées par un changement de coordonnées linéaire, il existe donc des coordonnées de classe $C^{1}$, définies sur un voisinage $U_{p}$ de $p$ dans lesquelles le champ $X$ s'écrit :

$$
X(x, y, z)=\sigma^{u} x \frac{\partial}{\partial x}-c_{p}\left(R+\alpha \frac{\partial}{\partial \theta}\right),
$$

avec $\sigma^{u}>0, \alpha \neq 0$, et $c_{p}>0$, où $R$ est le champ de vecteurs radial

$$
R(y, z)=y \frac{\partial}{\partial y}+z \frac{\partial}{\partial z} \quad \text { et } \quad \frac{\partial}{\partial \theta}=-z \frac{\partial}{\partial y}+y \frac{\partial}{\partial z}
$$

(par un abus de notation, on considère ces champs indifféremment comme des champs de 
$\mathbb{R}^{2}$ ou de $\mathbb{R}^{3}$ ). De plus, la connexion $\gamma$ coïncide, dans ces coordonnées, avec la demi-droite $x \geq 0, y=z=0$.

De même, il existe des coordonnées sur un voisinage $U_{q}$ de $q$ dans lesquelles $X$ s'écrit :

$$
X(x, y, z)=-\sigma^{s} x \frac{\partial}{\partial x}+c_{q}\left(R+\beta \frac{\partial}{\partial \theta}\right),
$$

avec $\sigma^{s}>0, \beta \neq 0$ et $c_{q}>0$. De plus la connexion $\gamma$ est la demi-droite $x \leq 0, y=z=0$.

On appelle alors $\alpha$ et $\beta$ les parties angulaires normalisées du champ $X$ aux points $p$ et $q$, respectivement. On note alors $X_{\alpha}$ et $X_{\beta}$ les champs de vecteurs de $\mathbb{R}^{2}$ définis par

$$
X_{\alpha}=R+\alpha \frac{\partial}{\partial \theta} \quad \text { et } \quad X_{\beta}=R+\beta \frac{\partial}{\partial \theta},
$$

et on les appelle les composantes transverses normalisées de la connexion $\gamma$. Si $M$ est orientée et si on impose aux coordonnées de préserver l'orientation alors les composantes transverses de $X$ le long de $\gamma$ sont canoniquement définies. Sinon, suivant le choix d'une orientation locale au voisinage de la connexion $\gamma$, les signes de $\alpha$ et $\beta$ peuvent varier (simultanément).

On considère la transition le long de la connexion $\gamma:$ soit $\Sigma_{p} \subset U_{p}$ le disque défini par $x=1$ et de même $\Sigma_{q} \subset U_{q}$ est le disque défini par $x=-1$. Ces disques coupent transversalement la connexion $\gamma$ chacun en un point $x_{p} \in U_{p}$ et $x_{q} \in U_{q}$, respectivement. Le champ $X$ induit une application $\mathcal{T}: \Sigma_{q} \rightarrow \Sigma_{p}$ définie au voisinage de $x_{p}$ et $x_{q}$, et appelée transition. Les coordonnées choisies sur $U_{p}$ et $U_{q}$ préservant l'orientation de $M$ (ou une orientation locale au voisinage de $\gamma$ ), la transition $\mathcal{T}$, exprimée dans les coordonnées $(y, z)$ de $\Sigma_{q}$ et $\Sigma_{p}$, préserve l'orientation.

On note $T$ la différentielle de la transition $\mathcal{T}$. L'application $T$ ainsi définie n'est pas unique : elle dépend des choix des coordonnées de linéarisation. On remarque que les homothéties et les rotations dans le plan des coordonnées $(y, z)$ laissent invariante l'expression des champs de vecteurs dans $U_{p}$ et $U_{q}$. On peut alors choisir des changements de coordonnées linéaires sur $U_{p}$ et $U_{q}$, par la composée d'une homothétie et d'une rotation dans les coordonnées $(y, z)$, de façon que la matrice $T$ s'écrive

$$
T=B_{\lambda}=\left(\begin{array}{ll}
1 & 0 \\
0 & \lambda
\end{array}\right), \quad \lambda \geq 1
$$

(dans les coordonnées $(y, z)$ des ouverts $U_{p}$ et $U_{q}$ restreintes à $\Sigma_{p}$ et $\Sigma_{q}$ ). On remarque que le nombre $\lambda \geq 1$ ainsi construit est indépendant des choix qui ont été faits. On note $t=\frac{1}{2}(\lambda+1 / \lambda)$, et on l'appellera le défaut de conformité de la transition.

1.2. Énoncé des résultats. On note $\psi:\left(\mathbb{R}^{*}\right)^{2} \rightarrow \mathbb{R}$ l'application définie par

$$
\psi(\alpha, \beta)=\frac{-\alpha \beta+|\alpha \beta| \sqrt{\left(\alpha^{2}+1\right)\left(\beta^{2}+1\right)}}{\alpha^{2} \beta^{2}} .
$$

Remarquons que $\psi(\alpha, \beta) \leq 1$ si et seulement si $\alpha=\beta \neq 0$, et alors $\psi(\alpha, \beta)=1$.

Considérons $U=\left\{(\alpha, \beta, t) \in \mathbb{R}^{3}, \alpha \neq 0, \beta \neq 0, t \geq \psi(x, y)\right.$ et $(\beta \neq \alpha$ ou $\left.t>1)\right\}$. Nous construisons une fonction $\Psi: U \rightarrow \mathbb{R}$, vérifiant les propriétés suivantes : 
- $\quad \Psi$ est continue sur $U$ et analytique sur $t>\psi(\alpha, \beta)$;

$-\quad \Psi(\alpha, \beta, t)=0 \Longleftrightarrow t=\psi(\alpha, \beta) ;$

- $\quad$ pour tous $\alpha, \beta$ fixés non-nuls, $\lim _{t \rightarrow+\infty}|\Psi(\alpha, \beta, t)|=+\infty$.

Nous montrons alors :

THÉORÈME 1.1. Deux champs de vecteurs $X$ et $\tilde{X}$ d'une variété de dimension 3 sont positivement topologiquement équivalents au voisinage de connexions $\gamma$ et $\tilde{\gamma}$ (entre des selles possédant des valeurs propres complexes) si et seulement si l'une des trois conditions suivantes est vérifiée :

(1) $\alpha=\beta, \tilde{\alpha}=\tilde{\beta}$ et $t=\tilde{t}=1$;

(2) $\quad(\beta-\alpha)(\tilde{\beta}-\tilde{\alpha})>0, t \leq \psi(\alpha, \beta)$ et $\tilde{t} \leq \psi(\tilde{\alpha}, \tilde{\beta})$;

(3) les conditions suivantes sont toutes vérifiées:
(a) $t>\psi(\alpha, \beta), \tilde{t}>\psi(\tilde{\alpha}, \tilde{\beta})$;
(b) $\alpha / \beta=\tilde{\alpha} / \tilde{\beta}$;
(c) $\Psi(\alpha, \beta, t)=\Psi(\tilde{\alpha}, \tilde{\beta}, \tilde{t})$;
(d) $\alpha \tilde{\alpha}>0$;

où $(\alpha, \beta, t)$ et $(\tilde{\alpha}, \tilde{\beta}, \tilde{t})$ sont les parties angulaires normalisées et le défaut de conformité des connexions $\gamma$ et $\tilde{\gamma}$.

Autrement dit, tant que le défaut de conformité $t$ de la transition est inférieur à $\psi(\alpha, \beta)$, le seul invariant de la connexion pour l'équivalence topologique (positive) est le signe de $\beta-\alpha$. Par contre, quand le défaut de conformité $t$ devient plus grand que $\psi(\alpha, \beta)$ alors le rapport $\alpha / \beta$ et $\Psi(\alpha, \beta, t)$ forment un système complet d'invariants pour l'équivalence topologique (il faut ajouter le signe de $\alpha$ pour l'équivalence positive).

Remarque 1.1. Si l'on n'exige pas que l'équivalence soit positive, il faut que l'une des 3 conditions du théorème soit vérifiée, soit pour le couple $((\alpha, \beta, t),(\tilde{\alpha}, \tilde{\beta}, \tilde{t}))$, soit pour le couple $((-\alpha,-\beta, t),(\tilde{\alpha}, \tilde{\beta}, \tilde{t}))$.

Voyons à présent les principales raisons de ce résultat :

Les composantes transverses normalisées $X_{\alpha}$ et $X_{\beta}$ du champ $X$ le long de la connexion $\gamma$ ne sont pas des invariants topologiques de cette connexion. Cependant considérons le couple $\left(X_{\alpha},\left(B_{\lambda}\right)_{*}\left(X_{\beta}\right)\right)$ de champs de vecteurs de $\mathbb{R}^{2}$, où $B_{\lambda}$ est la transition linéaire. Le théorème suivant montre que la classe d'équivalence topologique de ce couple est l'invariant complet de l'équivalence topologique de $X$ au voisinage de $\gamma$.

Plus précisément : pour tous $\alpha \neq 0, \beta \neq 0$ et $\lambda \geq 1$, notons $\mathcal{L}_{\alpha}$ le feuilletage singulier de $\mathbb{R}^{2}$ dirigé par $X_{\alpha}$ et $\mathcal{L}_{\beta}^{\lambda}$ le feuilletage singulier de $\mathbb{R}^{2}$ dirigé par $\left(B_{\lambda}\right)_{*}\left(X_{\beta}\right)$.

On dira que deux couples $\left(\mathcal{L}_{\alpha}, \mathcal{L}_{\beta}^{\lambda}\right)$ et $\left(\mathcal{L}_{\tilde{\alpha}}, \mathcal{L}_{\tilde{\beta}}^{\tilde{\lambda}}\right)$ sont topologiquement équivalents s'il existe un homéomorphisme $h$ de $\mathbb{R}^{2}$ tel que $h\left(\mathcal{L}_{\alpha}\right)=\mathcal{L}_{\tilde{\alpha}}$ et $h\left(\mathcal{L}_{\beta}^{\lambda}\right)=\mathcal{L}_{\tilde{\beta}}^{\tilde{\lambda}}$. Cette équivalence sera dite positive si $h$ préserve l'orientation.

On appellera couple caractéristique de la connexion $\gamma$, le couple de feuilletages $\left(\mathcal{L}_{\alpha}, \mathcal{L}_{\beta}^{\lambda}\right)$ où $X_{\alpha}$ et $X_{\beta}$ sont les composantes transverses normalisées de $X$ au voisinage de $p$ et $q$ et où $B_{\lambda}$ est la transition linéaire normalisée associée à la connexion $\gamma$.

Le Théorème 1.1 sera une conséquence du théorème suivant : 
THÉORÈME 1.2. Soient $X$ et $\tilde{X}$ deux champs de vecteurs de $\mathbb{R}^{3}$ présentant des connexions $\gamma$ et $\tilde{\gamma}$ entre des selles hyperboliques possédant des valeurs propres complexes, et soient $\left(\mathcal{L}_{\alpha}, \mathcal{L}_{\beta}^{\lambda}\right)$ et $\left(\mathcal{L}_{\tilde{\alpha}}, \mathcal{L}_{\tilde{\beta}}^{\tilde{\lambda}}\right)$ les couples caractéristiques des connexions $\gamma$ et $\tilde{\gamma}$.

Alors $X$ et $\tilde{X}$ sont (positivement) topologiquement équivalents au voisinage des connexions $\gamma$ et $\tilde{\gamma}$ si et seulement si les couples $\left(\mathcal{L}_{\alpha}, \mathcal{L}_{\beta}^{\lambda}\right)$ et $\left(\mathcal{L}_{\tilde{\alpha}}, \mathcal{L}_{\tilde{\beta}}^{\tilde{\lambda}}\right)$ sont (positivement) topologiquement équivalents.

Un simple calcul (voir le Lemme 3.2) montre que, si $t<\psi(\alpha, \beta)$, les champs de vecteurs $X_{\alpha}$ et $X_{\beta}^{\lambda}$ sont transverses en dehors de 0 . Si $\alpha \neq \beta$ et si $t=$ $\psi(\alpha, \beta)$ alors les deux champs sont tangents le long d'une unique droite, mais les feuilletages $\mathcal{L}_{\alpha}$ et $\mathcal{L}_{\beta}^{\lambda}$ sont encore topologiquement transverses. On montre alors que deux couples feuilletages (topologiquement) transverses $\left(\mathcal{L}_{\alpha}, \mathcal{L}_{\beta}^{\lambda}\right)$ et $\left(\mathcal{L}_{\tilde{\alpha}}, \mathcal{L}_{\tilde{\beta}}^{\lambda}\right)$ sont toujours topologiquement équivalents, l'équivalence étant positive ou négative suivant que l'orientation de $\mathbb{R}^{2}$ donnée par ' $\mathcal{L}_{\alpha}$ suivi de $\mathcal{L}_{\beta}^{\lambda}$ ' est égale ou opposée à celle donnée par ' $\mathcal{L}_{\tilde{\alpha}}$ suivi de $\mathcal{L}_{\tilde{\beta}}^{\tilde{\lambda}}$. Ceci explique l'item 2 du Théorème 1.1 : quand le défaut de conformité de la transition est assez petit, toutes les connexions (avec valeurs propres complexes) sont topologiquement équivalentes.

Si $t>\psi(\alpha, \beta)$ alors les feuilletages $\mathcal{L}_{\alpha}$ et $\mathcal{L}_{\beta}^{\lambda}$ possèdent deux droites de tangences $\Delta$ et $\Delta^{\prime}$. Le nombre $\alpha / \beta$ peut alors s'interpréter comme le nombre de translation relatif des holonomies $f$ et $g$ de ces feuilletages sur la droite $\Delta$. Le nombre $\Psi(\alpha, \beta, t)$ est alors le nombre de translation (relativement à $f$ ) de l'application de $\Delta$ obtenue comme la composée de l'holonomie de $\mathcal{L}_{\alpha}$ de $\Delta$ sur $\Delta^{\prime}$ par l'holonomie de $\mathcal{L}_{\beta}^{\lambda}$ de $\Delta^{\prime}$ sur $\Delta$ (nous verrons que ce nombre ne dépend que du choix d'un secteur délimité par les droites $\Delta$ et $\Delta^{\prime}$, et nous verrons comment faire canoniquement ce choix).

Ces nombres sont alors clairement des invariants d'équivalence topologique du couple $\left(\mathcal{L}_{\alpha}, \mathcal{L}_{\alpha}^{\lambda}\right)$, et on verra qu'ils forment un invariant complet pour cette équivalence. Ceci explique l'item 3 du Théorème $1.1:$ quand le défaut de conformité de la transition est grand $(t>\psi(\alpha, \beta))$, alors $(\alpha / \beta, \Psi(\alpha, \beta, t))$ est un invariant complet pour l'équivalence topologique des connexions de selles.

Dans la première partie de cet article nous allons introduire une relation d'équivalence (notée $\approx$ ) plus faible que l'équivalence topologique, entre les couples de feuilletages $\left(\mathcal{L}_{\alpha}, \mathcal{L}_{\beta}^{\lambda}\right)$ et $\left(\mathcal{L}_{\tilde{\alpha}}, \mathcal{L}_{\tilde{\beta}}^{\tilde{\lambda}}\right)$, et nous montrerons que la relation $\approx$ est équivalente à l'équivalence topologique des connexions $\gamma$ et $\tilde{\gamma}$.

La seconde partie donnera des conditions suffisantes à l'équivalence topologique (et donc a fortiori pour $\approx$ ) des couples $\left(\mathcal{L}_{\alpha}, \mathcal{L}_{\beta}^{\lambda}\right)$ et $\left(\mathcal{L}_{\tilde{\alpha}}, \mathcal{L}_{\tilde{\beta}}^{\tilde{\lambda}}\right)$.

La troisième partie montrera alors que ces conditions sont nécessaires à l'équivalence pour la relation $\approx$ (et donc aussi pour l'équivalence topologique) des couples $\left(\mathcal{L}_{\alpha}, \mathcal{L}_{\beta}^{\lambda}\right)$ et $\left(\mathcal{L}_{\tilde{\alpha}}, \mathcal{L}_{\tilde{\beta}}^{\tilde{\lambda}}\right)$.

Nous tenons à préciser que le referee de l'article a suggéré une preuve alternative, basée sur l'idée de la thèse de van Strien [8]. Cette preuve permet de raccourcir de manière significative les deux premières parties. Pour les conditions nécessaires (le point le plus délicat), l'argument développé ici nous semble incontournable et le point de vue adopté dans les deux premières parties prépare les résultats obtenus dans la troisième partie. 
2. Feuilletages de type radial et équivalence topologique au voisinage d'une selle

2.1. Définitions et relation d'équivalence. On appelle feuilletage radial de $\mathbb{R}^{2}$ le feuilletage singulier en $(0,0)$ dont les feuilles sont les demi-droites issues de $(0,0)$, ou sa restriction au disque $\left\{(x, y), x^{2}+y^{2} \leq 1\right\}$.

Soit $D \subset \mathbb{R}^{2}$ un disque topologique compact et $A$ un point de l'intérieur de $D$. Un feuilletage $\mathcal{F}$ de type radial sur le disque pointé $(D, A)$ est un feuilletage de $D$ possèdant un unique point singulier au point $A$, et qui est topologiquement équivalent au feuilletage radial. De même, un feuilletage du plan sera de type radial s'il est équivalent au feuilletage radial du plan.

Si $D$ est un disque d'un plan $P$, on peut prolonger tout feuilletage de type radial du disque $D$ en un feuilletage de type radial du plan $P$, topologiquement transverse à $\partial D$. De même, si $h$ est un homéomorphisme d'un disque (compact) $D$ sur une partie d'un disque $D^{\prime}$ et $\mathcal{F}$ un feuilletage de type radial sur $D$, alors on peut prolonger $h(\mathcal{F})$ en un feuilletage de type radial de $D^{\prime}$, (dont le point singulier est l'image par $h$ de celui de $\mathcal{F}$ ).

Si $\mathcal{F}$ est un feuilletage de type radial d'un disque $D$ on appellera secteur de $\mathcal{F}$ l'union $S_{I}$ des feuilles coupant un intervalle $I \subset \partial D$. Le secteur $S_{I}$ sera dit ouvert ou fermé suivant que l'intervalle $I$ sur lequel il s'appuie est lui-même ouvert ou fermé. On obtient ainsi une topologie naturelle sur l'espace des feuilles, donnée par celle de $\partial D$. La topologie donnée par un autre cercle transverse à $\mathcal{F}$ est équivalente à celle-ci. De même, pour un feuilletage de $\left(\mathbb{R}^{2}, A\right)$ de type radial, la topologie de l'espace des feuilles est donnée par un cercle transverse et est indépendante du choix de ce cercle.

Définition 2.1. Soient $\mathcal{F}$ et $\mathcal{F}^{\prime}$ deux feuilletages de type radial d'un disque pointé $(D, A)$.

(1) On dira que $\mathcal{F}$ guide $\mathcal{F}^{\prime}$ si pour tout recouvrement $\mathcal{U}$ de $D \backslash\{A\}$ par des secteurs ouverts de $\mathcal{F}$, il existe un voisinage $V$ de $A$ dans $D$ tel que pour toute feuille $L^{\prime}$ de $\mathcal{F}^{\prime}$ il existe un secteur $S \in \mathcal{U}$ de $\mathcal{F}$ tel que

$$
L^{\prime} \cap V \subset S
$$

(2) On dira que $\mathcal{F}$ est semblable à $\mathcal{F}^{\prime}$ et on notera $\mathcal{F} \simeq \mathcal{F}^{\prime}$ si $\mathcal{F}$ guide $\mathcal{F}^{\prime}$ et que $\mathcal{F}^{\prime}$ guide $\mathcal{F}$.

LEMME 2.1. La relation $\simeq$ est une relation d'équivalence.

Démonstration. Le seul point non trivial est la transitivité. Nous aurons besoin de montrer que si $\mathcal{F}$ guide les feuilles de $\mathcal{F}^{\prime}$ il guide aussi les secteurs de $\mathcal{F}^{\prime}$. Plus précisément :

Affirmation 2.1. Soient $\mathcal{F}, \mathcal{F}^{\prime}$ deux feuilletages de type radial de $(D, A)$ tels que $\mathcal{F}$ guide $\mathcal{F}^{\prime}$. Soit $\mathcal{U}$ un recouvrement de $D \backslash\{A\}$ par des secteurs ouverts de $\mathcal{F}$, alors il existe un recouvrement $\mathcal{U}^{\prime}$ de $D \backslash\{A\}$ par des secteurs ouverts de $\mathcal{F}^{\prime}$ et un voisinage $V$ de A tels que pour tout secteur $S^{\prime} \in \mathcal{U}^{\prime}$ il existe un secteur $S \in \mathcal{U}$ tel que $S^{\prime} \cap V \subset S$.

Démonstration. Soit $\mathcal{I}$ le recouvrement en intervalles ouverts de $\partial D$ induit par $\mathcal{U}$. On considère un recouvrement $\mathcal{J}$ de $\partial D$ par des intervalles ouverts, de façon que pour tout intervalle $J \in \mathcal{J}$ il existe un intervalle $I(J)$ de $\mathcal{I}$ qui contient tous les intervalles $J_{i} \in \mathcal{J}$ tels que $J_{i} \cap J \neq \emptyset$. On note $\mathcal{V}$ l'ensemble des secteurs $\left\{S_{J}\right\}_{J \in \mathcal{J}}$, pour le feuilletage $\mathcal{F}$. Comme $\mathcal{F}$ guide $\mathcal{F}^{\prime}$, il existe par définition un voisinage $V$ de $A$ tel que, pour toute feuille 
$L^{\prime}$ de $\mathcal{F}^{\prime}$, il existe un secteur $S_{J\left(L^{\prime}\right)} \in \mathcal{V}$ tel que $L^{\prime} \cap V \subset S_{J\left(L^{\prime}\right)}$. Notons $I\left(L^{\prime}\right)$ l'intervalle $I\left(J\left(L^{\prime}\right)\right)$ de $\mathcal{I}$.

Soit $L_{1}^{\prime}$ une feuille de $\mathcal{F}^{\prime}$ telle que $\left(L_{1}^{\prime} \cap V\right) \cap S_{J\left(L^{\prime}\right)} \neq \emptyset$. Alors $S_{J\left(L_{1}^{\prime}\right)} \cap S_{J\left(L^{\prime}\right)} \neq \emptyset$ donc $J\left(L_{1}^{\prime}\right) \cap J\left(L^{\prime}\right) \neq \emptyset$ et donc $J\left(L_{1}^{\prime}\right) \subset I\left(L^{\prime}\right)$ par définition de $I\left(L^{\prime}\right)$. On en déduit que le secteur $S_{I\left(L^{\prime}\right)}$ contient $L_{1}^{\prime} \cap V$, pour toute feuille $L_{1}^{\prime}$ de $\mathcal{F}^{\prime}$ rencontrant $S_{J\left(L^{\prime}\right)} \cap V$. L'union de ces feuilles est un voisinage de $L^{\prime}$ saturé pour $\mathcal{F}^{\prime}$ et donc contient un secteur ouvert $S\left(L^{\prime}\right)$ contenant la feuille $L^{\prime}$. L'ensemble des secteurs $S\left(L^{\prime}\right)$ forme le recouvrement annoncé.

Terminons maintenant la preuve du Lemme 2.1. Soit $\mathcal{F}^{\prime \prime}$ un feuilletage guidé par $\mathcal{F}^{\prime}$; soit $\mathcal{U}$ un recouvrement de $D \backslash\{A\}$ par des secteurs ouverts de $\mathcal{F}$ et soient $V$ le voisinage de $A$ et $\mathcal{U}^{\prime}$ le recouvrement par des secteurs ouverts de $\mathcal{F}^{\prime}$ donné par l'affirmation 2.1. Comme $\mathcal{F}^{\prime}$ guide $\mathcal{F}^{\prime \prime}$, il existe un voisinage $V^{\prime} \subset V$ de $A$ tel que pour toute feuille $L^{\prime \prime}$ de $\mathcal{F}^{\prime \prime}$, il existe un secteur $S^{\prime}$ de $\mathcal{U}^{\prime}$ contenant $L^{\prime \prime} \cap V^{\prime}$. Cependant $S^{\prime} \cap V$ est inclus dans un secteur $S$ de $\mathcal{U}$, qui contient a fortiori $L^{\prime \prime} \cap V^{\prime}$ : on vient de montrer que $\mathcal{F}$ guide $\mathcal{F}^{\prime \prime}$, ce qui conclut.

On montre alors facilement :

Lemme 2.2. Soient $\mathcal{F}$ et $\mathcal{F}^{\prime}$ deux feuilletages de type radial de $(D, A)$ qui sont semblables. Alors, pour toute feuille $L$ de $\mathcal{F}$, l'intersection des secteurs $S^{\prime}$ de $\mathcal{F}^{\prime}$ qui contiennent l'intersection de $L$ avec un voisinage de A (qui dépend de $S^{\prime}$ ) est exactement une feuille $L^{\prime}(L)$ du feuilletage $\mathcal{F}^{\prime}$. De plus l'application $L \mapsto L^{\prime}(L)$ est un homéomorphisme de l'espace des feuilles de $\mathcal{F}$ sur celui de $\mathcal{F}^{\prime}$.

2.2. Feuilletages adaptés et équivalence topologique au voisinage d'une selle. Soit $p$ un zéro hyperbolique de type selle d'un champ de vecteurs $X$ d'une variété de dimension 3 . On suppose, pour fixer les idées, que $W^{s}(p)$ est de dimension 2.

On fixe un voisinage $U$ sur lequel $X$ est topologiquement équivalent à un champ de vecteurs linéaire. Fixons aussi $W_{+}^{u}$ l'une des séparatrices instables locales de $p$, et $D$ un disque plongé dans $U$, transverse à $X$, coupant $W_{+}^{u}$ en un point $A$ et coupant toute orbite de $X$ sur $U$ en au plus un point. On choisit $U$ de façon que $U \backslash W_{\text {loc }}^{s}(p)$ possède deux composantes connexes, et on note $U_{+}$l'adhérence de la composante contenant la séparatrice locale $W_{+}^{u}$.

On appellera domaine fondamental de $W^{s}(p)$ tout cercle (continu) plongé dans $W_{\mathrm{loc}}^{s}(p)$ topologiquement transverse à la restriction de $X$ à $W^{s}(p)$, c'est-à-dire coupant chaque orbite de $W_{\mathrm{loc}}^{s}(p) \backslash\{p\}$ en un et un seul point.

On nommera couronne fondamentale tout plongement topologique $\mathcal{C}: S^{1} \times[0,1] \rightarrow$ $U_{+}$tel que $\mathcal{C}\left(S^{1} \times\{0\}\right)$ soit un domaine fondamental $C_{0}$ de $W^{S}(p)$ et coupant toute orbite de $X$ dans $U_{+}$en au plus un point, de façon topologiquement transverse. Chaque couronne fondamentale est munie du feuilletage vertical dont les feuilles sont les $\mathcal{C}(\{\theta\} \times[0,1])$, $\theta \in S^{1}$.

Remarquons que l'application de passage de coin $P$ (holonomie du champ de vecteurs $\left.X\right|_{U_{+}}$de $\mathcal{C}$ sur $D$ ) est bien définie de $\left.\left.\mathcal{C}\left(S^{1} \times\right] 0, \varepsilon\right]\right)$ sur $D \backslash\{A\}$, pour $\varepsilon>0$ assez petit.

Définition 2.2. On dira qu'un feuilletage $\mathcal{F}$ sur $D$ est un feuilletage adapté à $X$ si : 
(1) $\mathcal{F}$ est un feuilletage de type radial de $(D, A)$;

(2) Il existe une couronne fondamentale $\mathcal{C}$, telle que $P^{-1}(\mathcal{F})$ coïncide avec le feuilletage vertical sur $\left.\left.\mathcal{C}\left(S^{1} \times\right] 0,1\right]\right)$, où $P$ est l'holonomie de $X, \operatorname{de} \mathcal{C}$ sur $D$.

La définition d'un feuilletage adapté utilise l'existence d'une couronne fondamentale $\mathcal{C}$. Celle-ci n'est bien sûr pas unique, mais la notion de feuilletage adapté ne dépend pas de la couronne choisie. C'est ce qu'exprime la remarque ci-dessous :

Remarque 2.3. Soit $\mathcal{F}$ un feuilletage sur le disque pointé $(D, A)$ adapté à $X$ et $\mathcal{C}$ la couronne donnée par la définition. On se donne $\mathcal{C}^{\prime}: S^{1} \times[0,1] \rightarrow U_{+}$une autre couronne fondamentale. Alors il existe un homéomorphisme $h: S^{1} \times[0,1] \rightarrow S^{1} \times[0,1]$ avec la propriété suivante :

Notons $\mathcal{C}^{\prime \prime}$ la couronne fondamentale définie par $\mathcal{C}^{\prime \prime}=\mathcal{C}^{\prime}$ o $h$, et notons $P^{\prime \prime}$ l'application de passage de coin de $\mathcal{C}^{\prime \prime}$ sur $D$. Alors $\left(P^{\prime \prime}\right)^{-1}(\mathcal{F})$ coïncide avec le feuilletage vertical de $\left.\left.\mathcal{C}^{\prime \prime} \operatorname{sur} \mathcal{C}^{\prime \prime}\left(S^{1} \times\right] 0, \varepsilon\right]\right)$ pour $\varepsilon>0$ assez petit.

La notion de feuilletage adapté est bien déterminée, modulo la relation $\simeq$ :

Lemme 2.4. Soit $\mathcal{F}$ un feuilletage adapté à $X$. Un autre feuilletage de type radial $\mathcal{F}^{\prime}$ sur $(D, A)$ est adapté à $X$ si et seulement s'il est semblable à $\mathcal{F}$.

Démonstration. Soient $\mathcal{F}$ un feuilletage adapté, $\mathcal{C}: S^{1} \times[0,1] \rightarrow U_{+}$une couronne fondamentale donnée par la définition de feuilletage adapté, et $P$ l'application de passage de coin du champ $X$ de $\mathcal{C}$ sur $D$. Remarquons que $S$ est un secteur de $\mathcal{F}$ si et seulement s'il existe un intervalle $I$ de $S^{1}$ tel que $P^{-1}(S)$ est la bande $\mathcal{C}(I \times[0,1])$. On notera $S_{I}$ un tel secteur, et la longueur de $I$ sera l'amplitude du secteur. Montrons d'abord que tout feuilletage adapté $\mathcal{F}^{\prime}$ est semblable à $\mathcal{F}$. Soit $\mathcal{U}=\left\{S_{I}\right\}_{I \in \mathcal{I}}$ un recouvrement de $D \backslash\{A\}$ par des secteurs ouverts de $\mathcal{F}$ : la famille $\mathcal{I}$ d'intervalles de $S^{1}$ est un recouvrement de $S^{1}$ par des intervalles ouverts et on note $\varepsilon>0$ le nombre de Lebesgue de ce recouvrement. Notons $h: S^{1} \times[0,1] \rightarrow S^{1} \times[0,1]$ l'homéomorphisme donné par la Remarque 2.3. Par continuité uniforme de $h$, il existe $\delta_{1}>0$ tel que si $y \in\left[0, \delta_{1}\right]$ alors, pour tout $x \in S^{1}, h^{-1}(x, 0)$ et $h^{-1}(x, y)$ ont leurs projections sur $S^{1}$ qui diffèrent de moins de $\varepsilon$. Par définition de $\varepsilon$, il existe $I \in \mathcal{I}$ tel le segment $h^{-1}\left(\{x\} \times\left[0, \delta_{1}\right]\right)$ soit inclus dans la bande $I \times[0,1]$. Remarquons que, par définition de $h$, le feuilletage $P^{-1}\left(\mathcal{F}^{\prime}\right)$ coïncide avec l'image par $h^{-1}$ du feuilletage vertical de $\mathcal{C} \operatorname{sur} \mathcal{C}\left(S^{1} \times\left[0, \delta_{0}\right]\right)$ pour $\delta_{0}>0$ assez petit ; on choisit $\delta_{0}$ assez petit pour que la couronne $\mathcal{C}\left(S^{1} \times\left[0, \delta_{0}\right]\right)$ soit incluse dans l'image par $h^{-1}$ de la couronne $\mathcal{C}\left(S^{1} \times\left[0, \delta_{1}\right]\right)$.

On en déduit que, pour toute feuille $L^{\prime}$ de $\mathcal{F}^{\prime}$, l'intersection de $P^{-1}\left(L^{\prime}\right)$ avec $\mathcal{C}\left(S^{1} \times\right.$ $\left.\left[0, \delta_{0}\right]\right)$ est incluse dans une bande $I \times[0,1], I \in \mathcal{I}$. Notons $V=\{A\} \cup P\left(\mathcal{C}\left(S^{1} \times\left[0, \delta_{0}\right]\right)\right)$ : c'est un voisinage de $A$ dans $D$ et, pour toute feuille $L^{\prime}$ de $\mathcal{F}^{\prime}$, l'intersection $L^{\prime} \cap V$ est incluse dans un secteur $S_{I} \in \mathcal{U}$ ce qui montre que $\mathcal{F}$ guide $\mathcal{F}^{\prime}$. Le même argument permet de montrer que $\mathcal{F}^{\prime}$ guide $\mathcal{F}$.

Supposons à présent que $\mathcal{F}^{\prime}$ est un feuilletage semblable à $\mathcal{F}$. Si l'on note $C$ la couronne fondamentale associée à $\mathcal{F}$, il faut montrer que $P^{-1}\left(\mathcal{F}^{\prime}\right) \subset C \backslash C_{0}$ se prolonge en un feuilletage continu de $C$. 
Par définition, $\mathcal{F}^{\prime}$ est topologiquement équivalent au feuilletage radial : on peut donc considérer un système de coordonnées polaires continues $(r, \theta)$ sur $D$ pour lequel les feuilles de $\mathcal{F}^{\prime}$ soient les courbes $\{\theta=$ constante $\}$. Il reste donc à montrer que la fonction $P^{-1}(r, \theta)$ se prolonge par continuité en un homéomorphisme de $S^{1}$ sur $C_{0}$ quand $r$ tend vers 0 .

Montrons d'abord que pour toute feuille $L^{\prime}$ de $\mathcal{F}^{\prime}$, la feuille $P^{-1}\left(L^{\prime}\right)$ converge vers un point de $C_{0}$. Considérons une suite de secteurs $S_{n}$ du feuilletage $\mathcal{F}$ d'amplitude tendant vers 0 telle que chaque $S_{n}$ contienne un voisinage de $A$ dans $L^{\prime}$. Les secteurs $S_{n}$ ont comme image inverse $P^{-1}\left(S_{n}\right)$ qui est une bande s'appuyant sur un intervalle $J_{n}$ de $C_{0}$ et l'on vérifie que la longueur de $J_{n}$ tend vers 0 . La suite $J_{n}$ est décroissante pour l'inclusion donc l'intersection $\bigcap_{0}^{\infty} J_{n}$ est un point $x_{L^{\prime}}$, et on vérifie sans peine que $P^{-1}\left(L^{\prime}\right)$ converge vers $x_{L^{\prime}}$.

Soit $\left(r_{n}, \theta_{n}\right)$ une suite de points de $D$ telle que $r_{n}$ converge vers 0 et $\theta_{n}$ converge vers un point $\theta \in S^{1}$. Notons $L^{\prime}$ la feuille de $\mathcal{F}^{\prime}$ correspondant à $\theta$. Nous voulons montrer que $P^{-1}\left(r_{n}, \theta_{n}\right)$ converge vers $x_{L^{\prime}}$. Notons $L$ la feuille de $\mathcal{F}$ telle que $P^{-1}(L)$ se prolonge par $x_{L^{\prime}}$. Fixons $\varepsilon>0$ et notons $S_{\varepsilon}$ le secteur de $\mathcal{F}$ d'amplitude $\varepsilon$ autour de $L$. De l'affirmation 2.1 on peut déduire qu'il existe un voisinage $V$ de $A$ et un $\delta>0$ tel que $S^{\prime} \cap V$ soit inclus dans le secteur $S$, où $S^{\prime}$ est le secteur de $\mathcal{F}^{\prime}$ autour de $L$ et d'amplitude $\delta$.

Quand $\left|\theta_{n}-\theta\right|$ est assez petit, le point $\left(r_{n}, \theta_{n}\right)$ appartient au secteur $S^{\prime}$, de plus, quand $r_{n}$ est assez petit, ce point est dans $V$. On en déduit que $\left(r_{n}, \theta_{n}\right)$ est un point de $S_{\varepsilon}$. Le point $P^{-1}\left(r_{n}, \theta_{n}\right)$, pour $n$ grand, appartient à une bande arbitrairement étroite de feuilles de $P^{-1}(\mathcal{F})$ s'appuyant sur un petit intervalle autour de $x_{L^{\prime}}$ dans $C_{0}$ et la distance à $C_{0}$ tend vers 0 . Cette suite converge donc vers $x_{L^{\prime}}$.

Remarque 2.5. Si $\mathcal{F}$ et $\mathcal{F}^{\prime}$ sont deux feuilletages de $(D, A)$ adaptés à $X$, alors le Lemme 2.2 construit une bijection entre l'espace des feuilles de $\mathcal{F}$ et celui de $\mathcal{F}^{\prime}$. Cette bijection peut maintenant être vue de la façon suivante : à chaque feuille $L$ de $\mathcal{F}$, on associe la feuille $L^{\prime}$ de $\mathcal{F}^{\prime}$ telles que leurs images par $P^{-1}$ (sur une couronne fondamentale $\mathcal{C}$ ) aient le même point d'attache $\operatorname{sur} \mathcal{C}\left(S^{1} \times\{0\}\right)$.

L'intérêt principal de la notion de feuilletage adapté réside dans le lemme suivant :

LemMe 2.6. Soient p et $\tilde{p}$ deux zéros hyperboliques de champs $X$ et $\tilde{X}$, respectivement, sur des variétés de dimension 3, et dont la variété stable est de dimension 2 (le cas où la variété stable est de dimension un est analogue). Fixons $D$ et $\tilde{D}$ des disques transverses aux variétés instables locales $W_{\mathrm{loc}}^{u}(p)$ et $W_{\mathrm{loc}}^{u}(\tilde{p})$, en des points $A$ et $\tilde{A}$, respectivement. Soient $\mathcal{F}$ et $\tilde{\mathcal{F}}$ des feuilletages des disques $D$ et $\tilde{D}$ adaptés aux champs $X$ et $\tilde{X}$, respectivement. Finalement soit $h: D \rightarrow \tilde{D}$ un homéomorphisme tel que $h(A)=\tilde{A}$.

Alors les deux propriétés suivantes sont équivalentes :

- $\quad h$ se prolonge en une équivalence topologique entre les champs $X$ et $\tilde{X}$, d'un voisinage de p sur un voisinage de $\tilde{p}$;

- $\quad h(\mathcal{F})$ est semblable à $\tilde{\mathcal{F}}$.

Démonstration. Si $h$ se prolonge en une équivalence topologique $H: U \rightarrow \tilde{U}$ (où $U$ et $\tilde{U}$ sont des voisinages de $p$ et $\tilde{p}$ ) alors l'image par $H$ d'une couronne fondamentale $C \subset U$ de 


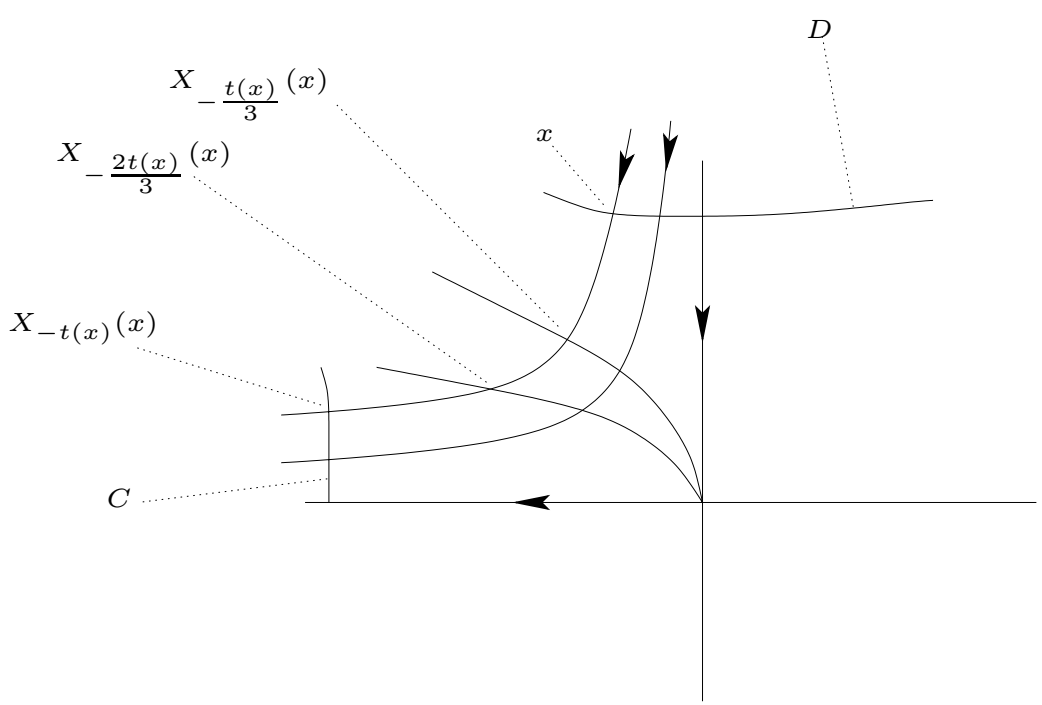

FIGURE 2. Les trois «cônes».

$X$ sera une couronne fondamentale de $\tilde{X}$, on en déduit aisément que $h(\mathcal{F})$ est un feuilletage adapté à $\tilde{X}$, donc semblable à $\tilde{\mathcal{F}}$, d'après le Lemme 2.4 .

Réciproquement, nous rappelons que sur une variété compacte $M$, deux champs $X$ et $\tilde{X}$ sont topologiquement équivalents si et seulement s'il existe un homéomorphisme $H$ de $M$, et une fonction $\sigma: M \times \mathbb{R} \rightarrow \mathbb{R}$ tels que $H\left(X_{t}(x)\right)=\tilde{X}_{\sigma(x, t)}(H(x))$ pour tout $x$ de $M$ et tout $t$ de $\mathbb{R}$.

L'idée est la suivante : au voisinage de la singularité, nous allons couper les orbites n'appartenant pas aux variétés invariantes en trois parties, en les intersectant avec trois «cônes». L'un des cônes contient la variété stable, un autre la variété instable et le troisième est entre les deux premiers (voir Figure 2). L'équivalence topologique est construite de sorte d'envoyer les cônes contenant les variétés invariantes de $p$ dans les cônes contenant les variétés invariantes de $\tilde{p}$. Ceci nous assure la continuité le long des variétés invariantes.

Fixons $C$ et $\tilde{C}$ des couronnes fondamentales de $X$ et de $\tilde{X}$. Pour tout point $x$ de $D$ suffisamment proche de $A$ son orbite négative coupe $C$ en $P^{-1}(x)$ et on note $t(x)>0$ le temps tel que $x=X_{t(x)}\left(P^{-1}(x)\right)$. On définit de même $\tilde{t}(\tilde{x})$ pour $\tilde{x} \in \tilde{D}$ proche de $\tilde{A}$.

On pose alors $\mu(x)=\frac{1}{3} \min (t(x), \tilde{t}(h(x)))$ (là où cela a un sens) et on définit $\sigma(x, t)$, $x \in D \backslash\{A\}, t \in[0,+\infty]$ de la façon suivante :

$$
\sigma(x, t)= \begin{cases}t & \text { si } t<\mu(x) \\ \tilde{t}(h(x))-t(x)+t & \text { si } t>t(x)-\mu(x) \\ \mu(x)+\frac{\tilde{t}(h(x))-2 \mu(x)}{t(x)-2 \mu(x)}(t-\mu(x)) & \text { si } \mu(x) \leq t \leq t(x)-\mu(x)\end{cases}
$$

et

$$
\sigma(A, t)=t
$$


La fonction $\sigma$ est continue : en un point $(x, t), x \neq A$, la continuité découle directement de la formule. D'autre part, pour tout $t$ et tout $x$ suffisamment proche de $A$, on a $\sigma(x, t)=t=\sigma(A, t)$, car les fonctions $t(x), \tilde{t}(h(x))$ et $\mu(x)$ sont continues et tendent vers l'infini quand $x$ tend vers $A$.

On définit $H$ par

$$
H\left(X_{-t}(x)\right)=\tilde{X}_{-\sigma(x, t)}(h(x)), \quad t \in[0,+\infty[.
$$

Remarquons d'abord que $H$ est un homéomorphisme de $U_{+} \backslash W_{\text {loc }}^{s}(p)$ sur $\tilde{U}_{+} \backslash W_{\text {loc }}^{s}(\tilde{p})$. Cela se déduit de la continuité de $\sigma$ et du fait que $\sigma(x, t(x))=\tilde{t}(h(x))$.

Nous laissons au lecteur le soin de vérifier la continuité de $H$ le long des variétés invariantes.

2.3. Équivalence topologique au voisinage d'une connexion. En corollaire du Lemme 2.6 on obtient une caractérisation de l'équivalence topologique de champs $X$ et $\tilde{X}$ au voisinage d'une connexion, en termes des couples de feuilletages obtenus sur un disque transverse à la connexion, à l'aide des deux singularités jointes par la connexion. Plus précisément :

Soit $X$ un champ de vecteurs d'une variété de dimension 3, présentant une connexion $\gamma$ entre deux selles hyperboliques $p$ et $q$ telle que $W^{u}(p)$ et $W^{s}(q)$ soient de dimension 1 . Notons $D$ un disque transverse à $X$ et coupant $\gamma$ en un unique point $A$. On appellera paire de feuilletages adaptée à la connexion $\gamma$ toute paire de feuilletages $(\mathcal{F}, \mathcal{G})$ de $D$ telle que $\mathcal{F}$ est adapté à la selle $p$ et $\mathcal{G}$ est adapté à la selle $q$. D'après le Lemme 2.4 si $\left(\mathcal{F}^{\prime}, \mathcal{G}^{\prime}\right)$ est une autre paire de feuilletages de $D$, adaptée à $\gamma$, alors $\mathcal{F} \simeq \mathcal{F}^{\prime}$ et $\mathcal{G} \simeq \mathcal{G}^{\prime}$.

Définition 2.3. Nous dirons que deux paires de feuilletages de type $\operatorname{radial}(\mathcal{F}, \mathcal{G})$ et $\left(\mathcal{F}^{\prime}, \mathcal{G}^{\prime}\right)$ sur des disques $D$ et $D^{\prime}$, respectivement, sont topologiquement similaires et nous noterons $(\mathcal{F}, \mathcal{G}) \approx\left(\mathcal{F}^{\prime}, \mathcal{G}^{\prime}\right)$ s'il existe un homéomorphisme $h: D \rightarrow D^{\prime}$ tel que $h(\mathcal{F}) \simeq \mathcal{F}^{\prime}$ et $h(\mathcal{G}) \simeq \mathcal{G}^{\prime}$.

On notera $(\mathcal{F}, \mathcal{G}) \approx_{+}\left(\mathcal{F}^{\prime}, \mathcal{G}^{\prime}\right)$ si l'homéomorphisme $h$ peut être choisi préservant l'orientation.

Corollaire 2.1. Soient $X$ et $\tilde{X}$ deux champs de vecteurs de variétés de dimension 3 , présentant chacun une connexion $\gamma$ et $\tilde{\gamma}$ de dimension 1 en des selles $(p, q)$ et $(\tilde{p}, \tilde{q})$ telles que $W^{u}(p, X), W^{u}(\tilde{p}, \tilde{X}), W^{s}(q, X)$ et $W^{s}(\tilde{q}, \tilde{X})$ sont de dimension 1. Soient $D$ et $\tilde{D}$ des disques transverses à $X$ et $\tilde{X}$ respectivement, et coupant chacun la connexion en un point A et $\tilde{A}$, respectivement. Soient $(\mathcal{F}, \mathcal{G})$ et $(\tilde{\mathcal{F}}, \tilde{\mathcal{G}})$ des paires de feuilletages de $D$ et $\tilde{D}$ adaptées à $\gamma$ et à $\tilde{\gamma}$, respectivement.

Les deux conditions suivantes sont équivalentes :

- les champs $X$ et $\tilde{X}$ sont topologiquement équivalents (respectivement positivement topologiquement équivalents) au voisinage des connexions $\gamma$ et $\tilde{\gamma}$;

- $\quad(\mathcal{F}, \mathcal{G}) \approx(\tilde{\mathcal{F}}, \tilde{\mathcal{G}})\left(\right.$ respectivement $\left.(\mathcal{F}, \mathcal{G}) \approx_{+}(\tilde{\mathcal{F}}, \tilde{\mathcal{G}})\right)$

Démonstration. S'il existe un homéomorphisme $h$ qui envoie un couple de feuilletages adaptés de $X$ pour les selles $p$ et $q$ sur un couple de feuilletages adaptés de $\tilde{X}$, alors le Lemme 2.6 permet de prolonger cet homéomorphisme en une équivalence topologique 
entre les champs. Cette équivalence préserve ou inverse l'orientation simultanément avec $h$.

Pour voir la réciproque, il suffit de remarquer que, s'il existe une équivalence topologique $H_{0}$ entre les champs $X$ et $\tilde{X}$ au voisinage de $\gamma$ et $\tilde{\gamma}$, alors il existe une autre équivalence $H_{1}$ qui induit un homéomorphisme d'un voisinage de $A$ dans $D$ sur un voisinage de $\tilde{A}$ dans $\tilde{D}$.

2.4. Feuilletages logarithmiques et difféomorphismes. On appelle feuilletage logarithmique de $\left(\mathbb{R}^{2}, 0\right)$ tout feuilletage de type radial invariant par les homothéties et les rotations de $\mathbb{R}^{2}$. Un tel feuilletage est dirigé par un champ de vecteurs du type

$$
X_{\mu}(x, y)=(x-\mu y) \frac{\partial}{\partial x}+(y+\mu x) \frac{\partial}{\partial y} ;
$$

un tel feuilletage est noté $\mathcal{L}_{\mu}$. Si $\mu=0$, il s'agit du feuilletage radial, et si $\mu \neq 0$ alors les feuilles de ce feuilletage sont des spirales logarithmiques. Dans toute la suite nous considérerons toujours $\mu \neq 0$.

On appellera encore feuilletages logarithmiques les images des feuilletages $\mathcal{L}_{\mu}$ par les applications linéaires inversibles $B \in G L(2, \mathbb{R})$, préservant l'orientation. Une telle application $B$ peut s'écrire de façon unique $B=B_{0} \circ B_{\lambda} \circ B_{1}$ où $B_{0}$ est une rotation, $B_{1}$ est la composée d'une homothétie et d'une rotation et où

$$
B_{\lambda}=\left(\begin{array}{ll}
1 & 0 \\
0 & \lambda
\end{array}\right), \quad \text { avec } \lambda \geq 1 .
$$

L'application $B_{1}$ préservant le feuilletage $\mathcal{L}_{\mu}$, le feuilletage $B\left(\mathcal{L}_{\mu}\right)$ est l'image par la rotation $B_{0}$ du feuilletage $\mathcal{L}_{\mu}^{\lambda}$ dirigé par le champ de vecteurs

$$
X_{\mu}^{\lambda}=\left(B_{\lambda}\right)_{*}\left(X_{\mu}\right)=\left(x-\frac{\mu}{\lambda} y\right) \frac{\partial}{\partial x}+(\lambda \mu x+y) \frac{\partial}{\partial y} .
$$

Remarque 2.7. Pour tout couple $(\mathcal{F}, \mathcal{G})$ de feuilletages logarithmiques, il existe un triplet $(\alpha, \beta, \lambda)$ avec $\lambda \geq 1$ et il existe $B \in S L(2, \mathbb{R})$ tel que $B(\mathcal{F})=\mathcal{L}_{\alpha}$ et $B(\mathcal{G})=\mathcal{L}_{\beta}^{\lambda}$.

Démonstration. Par définition il existe $B_{0}$ tel que $B_{0}(\mathcal{F})$ soit un feuilletage $\mathcal{L}_{\alpha}$. Il suffit de composer $B_{0}$ par une rotation (qui laisse invariante $\mathcal{L}_{\alpha}$ ) pour mettre $B_{0}(\mathcal{G})$ sous la forme $\mathcal{L}_{\beta}^{\lambda}$.

Le but de ce paragraphe est de montrer que l'image par un difféomorphisme, de la classe d'équivalence pour $\simeq$ d'un feuilletage logarithmique $\mathcal{F}$, est determinée par la différentielle en $(0,0)$ du difféomorphisme :

Proposition 2.1. Si $\mathcal{F}$ est un feuilletage logarithmique et si $\varphi: \mathbb{R}^{2} \rightarrow \mathbb{R}^{2}$ est un difféomorphisme ayant $(0,0)$ comme point fixe et tel que la différentielle de $\varphi$ en $(0,0)$ soit l'identité, alors

$$
\varphi(\mathcal{F}) \simeq \mathcal{F}
$$


LEMME 2.8. Soit $\mathcal{F}$ un feuilletage logarithmique de $\mathbb{R}^{2}$ et soit $\mathcal{U}$ un recouvrement de $\mathbb{R}^{2} \backslash\{(0,0)\}$ par des secteurs ouverts de $\mathcal{F}$. Alors il existe $\delta>0$ tel que, pour toute feuille $L$ de $\mathcal{F}$, il existe un secteur $S \in \mathcal{U}$ contenant toutes les boules $B(x,\|x\| \delta)$ où $x \in L$.

Démonstration. Remarquons d'abord que la propriété annoncée est préservée par l'action sur les feuilletages de $G L(2, \mathbb{R})$. On peut donc supposer que $\mathcal{F}$ est un feuilletage $\mathcal{L}_{\mu}$.

Comme $\mathcal{U}$ est un recouvrement par des ouverts et que le cercle $C_{1}=\left\{x \in \mathbb{R}^{2},\|x\|=1\right\}$ est compact on peut choisir $\delta>0$ tel que pour tout $x \in C_{1}$ la boule de centre $x$ et de rayon $\delta$ soit incluse dans un secteur $S(x) \in \mathcal{U}$.

Soit $L$ une feuille de $\mathcal{F}$; notons $x=L \cap C_{1}$, et $S$ le secteur $S(x)$. Soit $y$ un point de $L$. On considère le champ de vecteurs $X_{\mu}$ dirigeant le feuilletage $\mathcal{F}$. Il existe $t \in \mathbb{R}$ tel que $X_{\mu, t}(x)=y$. Remarquons que $X_{\mu, t}$ est la composée d'une homothétie et d'une rotation et donc que $X_{\mu, t}(B(x, \delta))=B(y,\|y\| \delta)$. De plus $X_{\mu, t}(S)=S$, et donc la $B(y,\|y\| \delta)$ est incluse dans $S$, ce qui conclut.

Nous pouvons à présent montrer la Proposition 2.1 :

Démonstration. Montrons que $\mathcal{F}$ guide le feuilletage $\varphi(\mathcal{F})$. Pour cela considérons un recouvrement $\mathcal{U}$ de $\mathbb{R}^{2} \backslash\{(0,0)\}$ par des secteurs ouverts de $\mathcal{F}$ et notons $\delta>0$ le nombre associé au recouvrement $\mathcal{U}$ par le Lemme 2.8. Pour toute feuille $L$ de $\mathcal{F}$ on notera $S(L) \in \mathcal{U}$ le secteur, donné par ce même lemme, tel qu'en tout point $y \in L$, la boule $B(y,\|y\| \delta)$ soit incluse dans $S(L)$.

Comme $\varphi$ est différentiable en $(0,0)$ et que sa différentielle en ce point est l'identité, il existe un voisinage $V$ de $(0,0)$ tel que pour tout point $y \in V$ on ait : $\|\varphi(y)-y\| \leq \delta / 2\|y\|$. On en déduit que $\varphi(L) \cap \varphi(V)$ est inclus dans $S(L)$, ce qui montre que $\mathcal{F}$ guide $\varphi(\mathcal{F})$.

Le même argument montre que $\mathcal{F}$ guide $\varphi^{-1}(\mathcal{F})$ et donc que $\varphi(\mathcal{F})$ guide $\mathcal{F}$. Nous avons bien montré que $\mathcal{F} \simeq \varphi(\mathcal{F})$.

2.5. Connexions entre selles ayant une valeur propre complexe. Soit $X$ un champ de vecteurs d'une variété de dimension 3, présentant une connexion $\gamma$ (de dimension 1) entre des selles $p$ et $q$ telles que $p$ ait une valeur propre complexe (non-réelle) de partie réelle négative et $q$ une valeur propre complexe de partie réelle positive. Soit $\left(\mathcal{L}_{\alpha}, \mathcal{L}_{\beta}^{\lambda}\right)$ le couple caractéristique de la connexion $\gamma$ (voir l'introduction, et l'énoncé du Théorème 1.2).

Remarque 2.9. Considérons les coordonnées de linéarisation (au voisinage de $p$ et de $q$ ) que nous avions fixées dans l'introduction, ainsi que les disques $\Sigma_{p}$ et $\Sigma_{q}$ transverses à $\gamma$. Le feuilletage $\mathcal{L}_{\alpha}$, considéré comme feuilletage de $\Sigma_{p}$ est alors adapté à la selle $p$, et de même le feuilletage $\mathcal{L}_{\beta}$ considéré comme feuilletage de $\Sigma_{q}$ est adapté à la selle $q$. Le feuilletage $\mathcal{L}_{\beta}^{\lambda}$, considéré comme feuilletage de $\Sigma_{p}$ est alors l'image de $\mathcal{L}_{\beta}$ par la différentielle de la transition : d'après la Proposition 2.1, il est donc semblable (pour $\simeq$ ) au feuilletage image de $\mathcal{L}_{\beta}$ par la transition, et donc est adapté à la singularité $q$.

Nous venons de voir que le couple caractéristique $\left(\mathcal{L}_{\alpha}, \mathcal{L}_{\beta}^{\lambda}\right)$ de $\gamma$ considéré comme paire de feuilletages de $\Sigma_{p}$ est une paire de feuilletages adaptée à la connexion $\gamma$.

En conséquence du Corollaire 2.1 on obtient : 
CoRollaire 2.2. Soient $X$ et $\tilde{X}$ deux champs de vecteurs possèdant chacun une connexion $\gamma$ et $\tilde{\gamma}$ entre des selles $p$ et $\tilde{p}$ (ayant une valeur propre contractante complexe) et $q$ et $\tilde{q}$ (ayant une valeur propre dilatante complexe). Notons $\left(\mathcal{L}_{\alpha}, \mathcal{L}_{\beta}^{\lambda}\right)$ et $\left(\mathcal{L}_{\tilde{\alpha}}, \mathcal{L}_{\tilde{\beta}}^{\tilde{\lambda}}\right)$ les couples caractéristiques des connexions $\gamma$ et $\tilde{\gamma}$.

Les deux propriétés suivantes sont équivalentes:

- $\quad X$ et $\tilde{X}$ sont topologiquement équivalents (respectivement positivement topologiquement équivalents) au voisinage des connexions $\gamma$ et $\tilde{\gamma}$;

$-\quad\left(\mathcal{L}_{\alpha}, \mathcal{L}_{\beta}^{\lambda}\right) \approx\left(\mathcal{L}_{\tilde{\alpha}}, \mathcal{L}_{\tilde{\beta}}^{\tilde{\lambda}}\right)\left(\right.$ respectivement $\left.\left(\mathcal{L}_{\alpha}, \mathcal{L}_{\beta}^{\lambda}\right) \approx_{+}\left(\mathcal{L}_{\tilde{\alpha}}, \mathcal{L}_{\tilde{\beta}}^{\tilde{\lambda}}\right)\right)$

3. Équivalence de couples de feuilletages logarithmiques : conditions suffisantes Rappelons que $\psi:\left(\mathbb{R}^{*}\right)^{2} \rightarrow \mathbb{R}$ est définie par

$$
\psi(\alpha, \beta)=\frac{-\alpha \beta+|\alpha \beta| \sqrt{\left(\alpha^{2}+1\right)\left(\beta^{2}+1\right)}}{\alpha^{2} \beta^{2}} .
$$

Considérons $U=\left\{(\alpha, \beta, t) \in \mathbb{R}^{3}, \alpha \neq 0, \beta \neq 0, t \geq \psi(x, y)\right.$ et $(\alpha \neq \beta$ ou $\left.t>1)\right\}$. Le but de cette partie est de construire l'application $\Psi: U \rightarrow \mathbb{R}$ annoncée pour le Théorème 1.1 et de montrer :

THÉORÈME 3.1. Deux couples $\left(\mathcal{L}_{\alpha}, \mathcal{L}_{\beta}^{\lambda}\right)$ et $\left(\mathcal{L}_{\tilde{\alpha}}, \mathcal{L}_{\tilde{\beta}}^{\tilde{\lambda}}\right)$ sont positivement topologiquement équivalents s'ils vérifient l'une des conditions suivantes :

(1) $\quad \alpha=\beta, \tilde{\alpha}=\tilde{\beta}$ et $t=\tilde{t}=1$;

(2) $\quad(\alpha-\beta)(\tilde{\alpha}-\tilde{\beta})>0, t \leq \psi(\alpha, \beta)$ et $\tilde{t} \leq \psi(\tilde{\alpha}, \tilde{\beta})$;

(3) les conditions suivantes sont toutes vérifiées:

(a) $t>\psi(\alpha, \beta), \tilde{t}>\psi(\tilde{\alpha}, \tilde{\beta})$;

(b) $\alpha / \beta=\tilde{\alpha} / \tilde{\beta}$;

(c) $\Psi(\alpha, \beta, t)=\Psi(\tilde{\alpha}, \tilde{\beta}, \tilde{t})$;

(d) $\alpha \tilde{\alpha}>0$;

où $t=\frac{1}{2}(\lambda+1 / \lambda)$ et $\tilde{t}=\frac{1}{2}(\tilde{\lambda}+1 / \tilde{\lambda})$.

Le Théorème 3.1 et le Corollaire 2.2 impliquent directement que les trois conditions du Théorème 1.1 sont des conditions suffisantes à l'équivalence topologique positive entre des connexions de selles.

3.1. Points de contact topologique et feuilletages logarithmiques. Soient $\sigma_{1}$ et $\sigma_{2}$ deux segments topologiques orientés plongés dans $\mathbb{R}^{2}$ (orienté). On suppose que les intérieurs de $\sigma_{1}$ et de $\sigma_{2}$ se coupent en un point $q$ isolé. Soit $V$ un voisinage de $q$ tel que $\sigma_{1}$ sépare $V$ en deux composantes connexes $V_{1}$ et $V_{2}$ telles que l'orientation de $\sigma_{1}$ coïncide avec celle dont il hérite comme bord de $V_{2}$. On dit que $q$ est un point d'intersection topologiquement transverse si tout voisinage de $q$ dans $\sigma_{2}$ rencontre $V_{1}$ et $V_{2}$. Nous dirons alors que $\sigma_{2}$ coupe $\sigma_{1}$ dans le sens positif s'il passe de $V_{1}$ à $V_{2}$, et négatif sinon : dans le cas où $\sigma_{1}$ et $\sigma_{2}$ sont des segments différentiables transverses en $q$, le segment $\sigma_{2}$ coupe $\sigma_{1}$ dans le sens positif si l'orientation, de la surface, obtenue en prenant l'orientation de $\sigma_{1}$ suivie de celle de $\sigma_{2}$ est positive. Le point $q$ est un point de tangence topologique s'il existe un voisinage de $q$ dans $\sigma_{2}$ qui est disjoint de $V_{1}$ ou de $V_{2}$. 
Les notions de tangence topologique ou d'intersection topologiquement transverse sont bien sûr préservées par l'action d'un homéomorphisme. Dans le cas d'une intersection transverse, l'orientation de l'intersection est préservée ou inversée suivant que l'homéomorphisme préserve ou inverse l'orientation.

Pour deux feuilletages du plan nous parlerons de points de tangence topologique ou d'intersection transverse, suivant que les feuilles passant par ce point se coupent de façon topologiquement tangente ou transversalement. Une courbe de tangences sera une courbe dont tous les points sont des points de tangence.

Deux feuilletages de type radial de $\left(\mathbb{R}^{2},(0,0)\right)$ seront dits topologiquement transverses s'ils n'ont aucun point de tangence dans $\mathbb{R}^{2} \backslash\{(0,0)\}$.

Remarque 3.1. Les feuilletages logarithmiques étant invariants par homothéties, tout point de tangence topologique entre deux feuilletages appartient à une droite de tangences entre ces feuilletages.

Le lemme suivant explique les trois cas intervenant dans l'énoncé des Théorèmes 1.1 et 3.1 :

LEMME 3.2. Étant donnés trois réels $\alpha \neq 0, \beta \neq 0$ et $\lambda \geq 1$, et $t=\frac{1}{2}(\lambda+1 / \lambda)$. On $a$ :

(1) si $\alpha=\beta$ et $t=1$, alors $\mathcal{L}_{\alpha}=\mathcal{L}_{\beta}^{\lambda}$;

(2) si $\alpha \neq \beta$ et $t \leq \psi(\alpha, \beta)$, les feuilletages $\mathcal{L}_{\alpha}$ et $\mathcal{L}_{\beta}^{\lambda}$ sont topologiquement transverses sur $\mathbb{R}^{2} \backslash\{(0,0)\}$;

(3) si $t>\psi(\alpha, \beta)$ il existe deux droites où les feuilletages $\mathcal{L}_{\alpha}$ et $\mathcal{L}_{\beta}^{\lambda}$ sont tangents et topologiquement tangents.

Démonstration. Les deux feuilletages étant différentiables, nous cherchons dans un premier temps les points de tangence différentiable ce qui amène à résoudre l'équation suivante :

$$
\left(x-\frac{\beta}{\lambda} y\right)(\alpha x+y)-(x-\alpha y)(\lambda \beta x+y)=0 .
$$

L'invariance par homothétie des feuilletages nous permet de restreindre l'étude de cette équation à la droite $\{y=1\}$ sur laquelle l'équation (1) s'écrit :

$$
(\alpha-\lambda \beta) x^{2}+\left(\alpha \beta \lambda-\frac{\alpha \beta}{\lambda}\right) x+\left(\alpha-\frac{\beta}{\lambda}\right)=0 .
$$

Le discriminant de cette équation en $x$ est un polynôme de degré deux en $t$ (pour $t \geq 1$ ) :

$$
\Delta(t)=4\left(\alpha^{2} \beta^{2} t^{2}+2 \alpha \beta t-\left(\alpha^{2}+\beta^{2}+\alpha^{2} \beta^{2}\right)\right) .
$$

Pour connaître le signe de $\Delta$, il faut résoudre l'équation $\Delta=0$ en $t$; on obtient un nouveau discriminant :

$$
\Delta^{\prime}=16 \alpha^{2} \beta^{2}\left(\alpha^{2}+1\right)\left(\beta^{2}+1\right) .
$$

On en déduit le résultat annoncé ; en effet comme par hypothèse le produit $\alpha \beta$ est nonnul, le discriminant $\Delta^{\prime}$ est strictement positif et donc l'équation $\Delta(t)=0$ admet deux solutions dans $\mathbb{R}$. Une seule de ces solutions est supérieure ou égale à 1 , elle s'écrit :

$$
t_{+}=\frac{-\alpha \beta+|\alpha \beta| \sqrt{\left(\alpha^{2}+1\right)\left(\beta^{2}+1\right)}}{\alpha^{2} \beta^{2}}=\psi(\alpha, \beta) .
$$

On conclut alors facilement. 
Le reste de cette section donne la démonstration du Théorème 3.1.

On remarque d'abord que le premier item du Théorème 3.1 est trivial : si $\mathcal{L}_{\alpha}=\mathcal{L}_{\beta}^{\lambda}$ et $\mathcal{L}_{\tilde{\alpha}}=\mathcal{L}_{\tilde{\beta}}^{\tilde{\lambda}}$ alors les deux couples $\left(\mathcal{L}_{\alpha}, \mathcal{L}_{\beta}^{\lambda}\right)$ et $\left(\mathcal{L}_{\tilde{\alpha}}, \mathcal{L}_{\tilde{\beta}}^{\tilde{\lambda}}\right)$ sont positivement topologiquement équivalents.

3.2. Équivalence topologique des couples de feuilletages logarithmiques transverses. Nous allons voir qu'en l'absence de droites de tangences (c'est-à-dire $\beta-\alpha \neq 0$ et $t \leq \psi(\alpha, \beta))$, il y a toujours un homéomorphisme envoyant un couple de feuilletages sur un autre. Ce résultat étant relativement classique, nous n'allons donner qu'une esquisse de la preuve :

LEMME 3.3. Fixons $\alpha \neq 0, \beta \neq 0, \tilde{\alpha} \neq 0, \tilde{\beta} \neq 0$ et $\lambda \geq 1, \tilde{\lambda} \geq 1$, tels que les feuilletages $\mathcal{L}_{\alpha}$ et $\mathcal{L}_{\beta}^{\lambda}$ soient topologiquement transverses sur $\mathbb{R}^{2} \backslash\{0\}$ et qu'il en est de même pour le couple $\left(\mathcal{L}_{\tilde{\alpha}}, \mathcal{L}_{\tilde{\beta}}^{\tilde{\lambda}}\right)$.

Alors il existe un homeomorphisme $h: \mathbb{R}^{2} \rightarrow \mathbb{R}^{2}$ tel que $h\left(\mathcal{L}_{\alpha}\right)=\mathcal{L}_{\tilde{\alpha}}$ et $h\left(\mathcal{L}_{\beta}^{\lambda}\right)=\mathcal{L}_{\tilde{\beta}}^{\tilde{\lambda}}$. Si de plus $(\beta-\alpha)(\tilde{\beta}-\tilde{\alpha})>0$ on peut choisir h préservant l'orientation.

Démonstration. (Esquisse) On considère les espaces de feuilles $C_{\alpha}$ et $C_{\beta}^{\lambda}$ des feuilletages $\mathcal{L}_{\tilde{\alpha}}$ et $\mathcal{L}_{\tilde{\beta}}^{\tilde{\lambda}}$ respectivement (en orientant les feuilles «fuyant l'origine», on induit une orientation sur les cercles $C_{\alpha}$ et $C_{\beta}^{\lambda}$ ). Le produit $C_{\alpha} \times C_{\beta}^{\lambda}$ est muni des deux feuilletages en cercles triviaux, et l'application $\pi: \mathbb{R}^{2} \backslash\{(0,0)\} \rightarrow C_{\alpha} \times C_{\beta}^{\lambda}$ est un revêtement (infini) envoyant feuilletages sur feuilletages. On considère aussi le revêtement $\tilde{\pi}$ associé au couple $\left(\mathcal{L}_{\tilde{\alpha}}, \mathcal{L}_{\tilde{\beta}}^{\tilde{\lambda}}\right)$. Un homémorphisme $\phi$, de $C_{\alpha} \times C_{\beta}^{\lambda}$ vers $C_{\tilde{\alpha}} \times C_{\tilde{\beta}}^{\tilde{\lambda}}$ se relevant à $\mathbb{R}^{2} \backslash\{(0,0)\}$ va conjuguer les couples $\left(\mathcal{L}_{\tilde{\alpha}}, \mathcal{L}_{\tilde{\beta}}^{\tilde{\lambda}}\right)$ et $\left(\mathcal{L}_{\tilde{\alpha}}, \mathcal{L}_{\tilde{\beta}}^{\tilde{\lambda}}\right)$ s'il s'écrit $\phi=\left(\varphi_{1}, \varphi_{2}\right)$ avec $\varphi_{1}: C_{\alpha} \rightarrow C_{\tilde{\alpha}}$ et $\varphi_{2}: C_{\beta}^{\lambda} \rightarrow C_{\tilde{\beta}}^{\tilde{\lambda}}$ deux homéomorphismes préservant ou inversant simultanément l'orientation. On montre facilement :

AfFirmation 3.1. Quand $\beta-\alpha>0$, les feuilles (orientées) de $\mathcal{L}_{\alpha}$ parcourent l'espace $C_{\beta}^{\lambda}$ dans le sens négatif, alors que les feuilles de $\mathcal{L}_{\beta}^{\lambda}$ parcourent $C_{\alpha}$ dans le sens direct, les orientations étant inversées si $\beta-\alpha<0$.

On termine la preuve du lemme en choisissant, quand $(\beta-\alpha)(\tilde{\beta}-\tilde{\alpha})>0, \varphi_{1}: C_{\alpha} \rightarrow C_{\tilde{\alpha}}$ et $\varphi_{2}: C_{\beta}^{\lambda} \rightarrow C_{\tilde{\beta}}^{\tilde{\lambda}}$ deux homéomorphismes préservant les orientations. Alors $\phi=\left(\varphi_{1}, \varphi_{2}\right)$ est un homéomorphisme préservant les feuilletages en cercles munis de leurs orientations. $\phi$ admet des relèvements $\Phi: \mathbb{R}^{2} \backslash\{(0,0)\} \rightarrow \mathbb{R}^{2} \backslash\{(0,0)\}$, et tout relèvement conjugue le feuilletage $\mathcal{L}_{\alpha}$ à $\mathcal{L}_{\tilde{\alpha}}$ et le feuilletage $\mathcal{L}_{\beta}^{\lambda}$ à $\mathcal{L}_{\tilde{\beta}}^{\tilde{\lambda}}$. De l'affirmation, on déduit que $\Phi$ est l'homéomorphisme annoncé : il préserve l'orientation des feuilles de $\mathcal{L}_{\alpha}$ et de $\mathcal{L}_{\tilde{\alpha}}$ (donc se prolonge par continuité en $(0,0))$, et préserve l'orientation de $\mathbb{R}^{2}$.

Quand $(\beta-\alpha)(\tilde{\beta}-\tilde{\alpha})<0$, on choisit $\varphi_{1}$ et $\varphi_{2}$ renversant l'orientation pour arriver à la conclusion.

3.3. Couples de feuilletages logarithmiques ayant deux droites de tangences : conditions suffisantes à l'équivalence topologique. Soient $\alpha \neq 0, \beta \neq 0$ et $\lambda>1$ tels que 
$t=\frac{1}{2}(\lambda+1 / \lambda)$ soit strictement supérieur à $\psi(\alpha, \beta)$. D'après le Lemme 3.2, les feuilletages $\mathcal{L}_{\alpha}$ et $\mathcal{L}_{\beta}^{\lambda}$ sont tangents le long de deux droites, qui coupent le plan $\mathbb{R}^{2}$ en deux cônes. Remarquons que la base $\left(X_{\alpha}, X_{\beta}^{\lambda}\right)$ est alternativement directe ou indirecte suivant les cônes et on note $S_{0}$ celui pour lequel le déterminant de $\left(X_{\alpha}, X_{\beta}^{\lambda}\right)$, écrit dans la base directe $(R, \partial / \partial \theta)$, soit de signe contraire à $\beta-\alpha:$ quand $\alpha \neq \beta$ et que $t$ tend vers $\psi(\alpha, \beta)$ ce cône devient petit et dégénère en la droite de tangences. On note alors $\Delta$ et $\Delta^{\prime}$ les droites de tangences du couple $\left(\mathcal{L}_{\alpha}, \mathcal{L}_{\beta}^{\lambda}\right)$ de façon que l'arc de cercle unité traversant $S_{0}$ et orienté dans le sens trigonométrique direct ait son origine sur $\Delta$ et son extrémité sur $\Delta^{\prime}$.

Notons $f: \Delta \rightarrow \Delta^{\prime}$ et $g: \Delta \rightarrow \Delta^{\prime}$ les holonomies des restrictions à $S_{0}$ des feuilletages $\mathcal{L}_{\alpha}$ et $\mathcal{L}_{\beta}^{\lambda}$, respectivement. Ce sont des applications linéaires. La composée $H=g^{-1} \circ f: \Delta \rightarrow \Delta$ est alors une homothétie de $\Delta$. Notons $\mu(\alpha, \beta, t)>0$ le rapport de cette homothétie.

Voyons quelques propriétés de la fonction $\mu$ :

- $\quad \mu$ est une fonction continue de $\alpha, \beta, t$, pour $t>\psi(\alpha, \beta)$;

- $\quad \mu(\alpha, \beta, t)$ est toujours différent de 1 : cela découle du fait que les feuilletages sont transverses dans l'intérieur du secteur $S_{0}$;

- la fonction $\mu$ est analytique : en effet les droites de tangences dépendent analytiquement de $\alpha, \beta, \lambda$ et l'holonomie de ces feuilletages d'une droite sur une autre dépend analytiquement des droites considérées ;

- $\quad$ si $\alpha \neq \beta$ et que $t$ tend vers $\psi(\alpha, \beta)$ chacune des holonomies $f$ et $g$ converge vers l'identité de la droite de tangences, ce qui montre que $\mu$ se prolonge par continuité par 1 sur l'ensemble $\alpha \neq \beta$ et $t=\psi(\alpha, \beta)$;

- $\quad$ on vérifie que, quand $t$ tend vers l'infini, $\mu(\alpha, \beta, t)$ tend vers 0 ou $+\infty$ : en effet les droites de tangences de $\mathcal{L}_{\alpha}$ et $\mathcal{L}_{\beta}^{\lambda}$ convergent vers deux droites transverses et l'holonomie $g$ de $\mathcal{L}_{\beta}^{\lambda}$ converge vers une contraction ou une dilatation de rapport infini.

On note $\Psi(\alpha, \beta, t)=(\alpha / 2 \pi) \log (\mu(\alpha, \beta, t))$. Les propriétés annoncées pour les Théorèmes 1.1 et 3.1 découlent directement de celles de $\mu$.

Le but de ce paragraphe est de montrer la proposition suivante, qui achève la démonstration du Théorème 3.1 :

Proposition 3.1. Soient $(\alpha, \beta, \lambda)$ et $(\tilde{\alpha}, \tilde{\beta}, \tilde{\lambda})$ deux triplets tels que $\alpha, \beta, \tilde{\alpha}, \tilde{\beta}$ sont nonnuls, $t=\frac{1}{2}(\lambda+1 / \lambda)>\psi(\alpha, \beta)$ et que $\tilde{t}=\frac{1}{2}(\tilde{\lambda}+1 / \tilde{\lambda})>\psi(\tilde{\alpha}, \tilde{\beta})$.

Supposons que $\alpha / \beta=\tilde{\alpha} / \tilde{\beta}$ et $\Psi(\alpha, \beta, t)=\Psi(\tilde{\alpha}, \tilde{\beta}, \tilde{t})$ et que $\alpha$ et $\tilde{\alpha}$ sont de même signe.

Alors il existe un homéomorphisme $h$ de $\mathbb{R}^{2}$, préservant l'orientation, tel que $h\left(\mathcal{L}_{\alpha}\right)=$ $\mathcal{L}_{\tilde{\alpha}}$ et $h\left(\mathcal{L}_{\beta}^{\lambda}\right)=\mathcal{L}_{\tilde{\beta}}^{\tilde{\lambda}}$.

Démonstration (Esquisse) On note $\mathcal{I}$ la symétrie $x \mapsto-x$ de $\Delta$ et $D_{\alpha}$ et $D_{\beta}$ les applications de premier retour sur la droite $\Delta$ des feuilletages $\mathcal{L}_{\alpha}$ et $\mathcal{L}_{\beta}^{\lambda}$ dont les feuilles sont parcourues en suivant le sens trigonométrique direct (ceci a un sens puisque ces feuilletages sont transverses au radial). On vérifie que $D_{\alpha}$ est l'homothétie de rapport $-e^{\pi / \alpha}$ et $D_{\beta}$ est l'homothétie de rapport $-e^{\pi / \beta}$. Rappelons que $H=g^{-1} \circ f$ est une homothétie de $\Delta$ de rapport $\mu$. 
On définit de même le cône $\tilde{S}_{0}$, les droites $\tilde{\Delta}$ et $\tilde{\Delta}^{\prime}$, et les applications $D_{\tilde{\alpha}}, D_{\tilde{\beta}}, \tilde{H}$ et $\tilde{\mathcal{I}}$ de la droite $\tilde{\Delta}$, qui sont des homothéties de rapport $-e^{\pi / \tilde{\alpha}},-e^{\pi / \tilde{\beta}}, \tilde{\mu}=\mu(\tilde{\alpha}, \tilde{\beta}, \tilde{t})$ et -1 respectivement.

LEMME 3.4. Il existe un homéomorphisme $h_{0}: \Delta \rightarrow \tilde{\Delta}$ conjuguant $D_{\alpha}, D_{\beta}, H$ et $\mathcal{I} \grave{a}$ $D_{\tilde{\alpha}}, D_{\tilde{\beta}}, \tilde{H}$ et $\tilde{\mathcal{I}}$, respectivement.

Démonstration. En appliquant un homéomorphisme de type «logarithme», on peut conjuguer les applications de retour des feuilletages sur $\Delta$ à des translations. Le lemme est alors une conséquence des hypothèses $\tilde{\alpha} / \tilde{\beta}=\alpha / \beta$ et $\Psi(\alpha, \beta, t)=\Psi(\tilde{\alpha}, \tilde{\beta}, \tilde{t})$ qui entrainent l'égalité des nombres de translation des applications de retour.

Reprenons la preuve de la proposition : nous allons construire, dans un premier temps, l'homéomorphisme de conjugaison $h$ du cône $S_{0}$ sur le cône $\tilde{S}_{0}$.

Soit $x \in S_{0} \backslash\{(0,0)\}$. On considère $\gamma_{x}$ et $\gamma_{x}^{\prime}$ les segments de feuilles de $\mathcal{F}$ et $\mathcal{G}$ passant par $x$, d'origine sur $\Delta$ et d'extrémité sur $\Delta^{\prime}$. Notons $y$ l'origine de $\gamma_{x}$ et $z$ celle de $\gamma_{x}^{\prime}$. On vérifie facilement que les points $y$ et $z$ varient continûment en fontion de $x$, et qu'ils coïncindent si et seulement si $x \in \Delta$ (ils coïncindent alors avec $x$ ). Soient $\tilde{y}=h_{0}(y) \in \tilde{\Delta}$ et $\tilde{z}=h_{0}(z) \in \tilde{\Delta}$. Notons $\tilde{\gamma}$ et $\tilde{\gamma}^{\prime}$ les segments de feuilles de $\tilde{\mathcal{F}}$ et $\tilde{\mathcal{G}}$ d'origine $\tilde{y}$ et $\tilde{z}$, contenus dans le secteur $\tilde{S}_{0}$.

AfFirmation 3.2. Les segments $\tilde{\gamma}$ et $\tilde{\gamma}^{\prime}$ se coupent en un et un seul point $h(x) \in \tilde{S}_{0}$.

Démonstration. Remarquons que les segments de feuilles se coupent en au plus un point dans $S_{0}$. Nous devons donc montrer que $\tilde{\gamma} \cap \tilde{\gamma}^{\prime}$ n'est pas vide. On se convainc facilement qu'il suffit de montrer que $\tilde{z}$ appartient au segment de $\tilde{\Delta}$ joignant $\tilde{y}$ à $\tilde{H}(\tilde{y})$. On remarque que ce segment est l'image par $h_{0}$ du segment joignant $y$ à $H(y)$, car $h_{0}$ conjugue $H$ à $\tilde{H}$. Le point $z$ appartient au segment de $\Delta$ joignant $y$ à $H(y)$, ce qui conclut.

On a ainsi défini une application $h: S_{0} \rightarrow \tilde{S}_{0}$ par $x \mapsto h(x)$. En utilisant la transversalité des feuilletages dans l'intérieur de $S_{0}$ on se convainc que $h$ est continu. D'autre part on peut construire l'inverse de $h$, par le même procédé : $h$ est donc un homéomorphisme de $S_{0}$ sur $\tilde{S}_{0}$, qui coïncide avec $h_{0}$ en restriction à $\Delta$. Notons $h_{0}^{\prime}$ la restriction de $h$ à la droite $\Delta^{\prime}$.

Nous devons à présent étendre $h$ aux cônes $S_{1}$ et $\tilde{S}_{1}$, complémentaires de $S_{0}$ et $\tilde{S}_{0}$. Pour cela nous remarquons que $h_{0}^{\prime}$ joue, pour le secteur $S_{1}$, le même rôle que $h_{0}$ pour le secteur $S_{0}$ : par construction, il conjugue les applications de retour sur $\Delta^{\prime}$. On procède alors de même que dans $S_{0}$ et $\tilde{S}_{0}$ en vérifiant que le recollement se fait de manière continue le long de $\Delta$.

\section{Conditions nécessaires à la similarité des couples de feuilletages logarithmiques}

Le but de cette section est d'achever la démonstration du Théorème 1.1, en montrant que les conditions suffisantes à l'équivalence topologique de couples $\left(\mathcal{L}_{\alpha}, \mathcal{L}_{\beta}^{\lambda}\right)$ et $\left(\mathcal{L}_{\tilde{\alpha}}, \mathcal{L}_{\tilde{\beta}}^{\tilde{\lambda}}\right)$, exhibées dans la section précédente, sont en fait des conditions nécessaires (et suffisantes) pour que $\left(\mathcal{L}_{\alpha}, \mathcal{L}_{\beta}^{\lambda}\right) \approx_{+}\left(\mathcal{L}_{\tilde{\alpha}}, \mathcal{L}_{\tilde{\beta}}^{\tilde{\hat{\lambda}}}\right)$. 
Soient deux couples $\left(\mathcal{L}_{\alpha}, \mathcal{L}_{\beta}^{\lambda}\right)$ et $\left(\mathcal{L}_{\tilde{\alpha}}, \mathcal{L}_{\tilde{\beta}}^{\tilde{\lambda}}\right)$ et un homéomorphisme $h$ de $\mathbb{R}^{2}$, préservant l'orientation, tel que $h\left(\mathcal{L}_{\tilde{\alpha}}\right) \simeq \mathcal{L}_{\alpha}$ et $h\left(\mathcal{L}_{\tilde{\beta}}^{\tilde{\lambda}}\right) \simeq \mathcal{L}_{\beta}^{\lambda}$.

Remarquons d'abord que, si $\mathcal{L}_{\alpha}=\mathcal{L}_{\beta}^{\lambda}$ (c'est-à-dire si $\alpha=\beta \neq 0$ et $\lambda=1$ ), alors les feuilletages $\mathcal{L}_{\tilde{\alpha}}$ et $\mathcal{L}_{\tilde{\beta}}^{\tilde{\lambda}}$ sont semblables. On vérifie facilement :

Lemme 4.1. Deux feuilletages $\mathcal{L}_{\tilde{\alpha}}$ et $\mathcal{L}_{\tilde{\beta}}^{\tilde{\lambda}}$ sont semblables si et seulement s'ils sont confondus.

Le Théorème 1.1 est à présent une conséquence directe de la proposition suivante :

Proposition 4.1. On suppose que les feuilletages $\mathcal{L}_{\alpha}$ et $\mathcal{L}_{\beta}^{\lambda}$ sont tangents le long de deux droites. Alors :

(1) les feuilletages $\mathcal{L}_{\tilde{\alpha}}$ et $\mathcal{L}_{\tilde{\beta}}^{\tilde{\lambda}}$ sont tangents le long de deux droites ;

(2) $\alpha \tilde{\alpha}>0$;

(3) $\alpha / \beta=\tilde{\alpha} / \tilde{\beta}$;

(4) $\Psi(\alpha, \beta)=\Psi(\tilde{\alpha}, \tilde{\beta})$.

La preuve de cette proposition est le but de cette section. Notre premier but est donc de prouver l'invariance du nombre de droites de tangences entre deux feuilletages $\operatorname{logarithmiques~sous~la~relation~de~similarité~}(\approx)$. De plus, afin de préparer les autres points de la preuve, nous avons besoin de montrer que si $\mathcal{L}_{\alpha}$ est équivalent à $h\left(\mathcal{L}_{\tilde{\alpha}}\right)$ et que $\mathcal{L}_{\beta}^{\lambda}$ est équivalent à $h\left(\mathcal{L}_{\tilde{\beta}}^{\tilde{\lambda}}\right)$ alors l'image par $h$ des droites de tangences des feuilletages $\mathcal{L}_{\tilde{\alpha}}$ et $\mathcal{L}_{\tilde{\beta}}^{\tilde{\lambda}}$ sont des courbes $\left(C^{0}\right)$ qui sont tangentes en $(0,0)$ aux droites de tangences des feuilletages $\mathcal{L}_{\alpha}$ et $\mathcal{L}_{\beta}^{\lambda}$

Pour cela nous allons devoir construire une structure, appelée ici chapelet de perles, qui piège les images par $h$ des droites de tangences.

Dans toute cette section, si $x$ et $y$ sont deux points d'une même feuille d'un feuilletage de type radial $\mathcal{F}$, on notera $[x, y]_{\mathcal{F}}$ le segment de feuille qui les joint.

\subsection{Bigones et chapelets de perles.}

Définition 4.1. Soient $\mathcal{F}$ et $\mathcal{G}$ deux feuilletages de type radial en $(0,0)$. Un bigone est un disque $\Delta$ ne contenant pas $(0,0)$, dont le bord est constitué d'un segment $\partial_{\mathcal{F}}(\Delta)$ de feuille de $\mathcal{F}$ et d'un segment $\partial_{\mathcal{G}}(\Delta)$ de feuille de $\mathcal{G}$.

Un coin d'un bigone est un point de $\partial_{\mathcal{F}}(\Delta) \cap \partial_{\mathcal{G}}(\Delta)$.

Lemme 4.2. Soient $\alpha \neq 0, \beta \neq 0$ et $\lambda \geq 1$. Pour tout bigone $\Delta$ du couple $\left(\mathcal{L}_{\alpha}, \mathcal{L}_{\beta}^{\lambda}\right)$, les points de tangence entre $\mathcal{L}_{\alpha}$ et $\mathcal{L}_{\beta}^{\lambda}$ dans $\Delta$ privé de ses coins forment un segment de droite joignant $\operatorname{int}\left(\partial_{\mathcal{L}_{\alpha}}(\Delta)\right) \grave{a} \operatorname{int}\left(\partial_{\mathcal{L}_{\beta}^{\lambda}}(\Delta)\right)$.

Démonstration. Afin d'alléger les notations, nous noterons $\mathcal{F}=\mathcal{L}_{\alpha}$ et $\mathcal{G}=\mathcal{L}_{\beta}^{\lambda}$. On vérifie facilement que l'existence de bigone implique l'existence de points où les feuilletages $\mathcal{F}$ et $\mathcal{G}$ ne sont pas topologiquement transverses. Ils possèdent donc exactement deux droites de tangences. Pour une feuille $F$ de $\mathcal{F}$, nous numéroterons $F_{n}$, avec $n$ dans $\mathbb{Z}$, les segments de $F$ compris entre deux demi-droites de tangences en suivant le sens trigonométrique. 


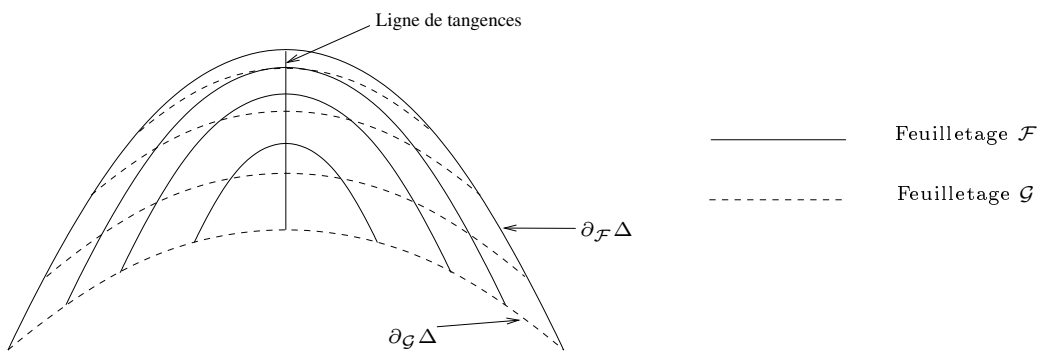

FIGURE 3. Une perle et ses feuilletages.

Nous agirons de même pour le feuilletage $\mathcal{G}$. Nous remarquons que deux morceaux de feuilles $F_{n}$ et $G_{m}$ ne peuvent se rencontrer dans l'intérieur d'un demi-cône qu'en au plus un point.

On considère $F_{0}$ et $G_{0}$ deux segments de feuilles de $\mathcal{F}$ et $\mathcal{G}$ respectivement, dans un même demi-cône. Le lemme est une conséquence de l'affirmation suivante :

AfFirmation 4.1. Si $F_{0}$ et $G_{0}$ ont une intersection non vide, alors soit $F_{1}$ et $G_{1}$ ont une intersection non vide, soit $F_{n}$ et $G_{m}$ pour $n$ et $m$ strictement positifs ne se rencontrent pas.

Démonstration. Supposons que $F_{1}$ et $G_{1}$ ne se rencontrent pas. Dans l'intérieur de chaque demi-cône, l'orientation de $\mathcal{F}$ suivi de $\mathcal{G}$ est constante et cette orientation change lorsque l'on passe d'un demi-cône à un demi-cône adjacent. On en déduit que $F_{2}$ et $G_{2}$ ne se rencontrent pas. Grâce au même argument, on montre que le long de la demi-droite de tangences entre $F_{1}$ et $F_{2}$, si le point $F_{1} \cap F_{2}$ est plus près de l'origine que le point $G_{1} \cap G_{2}$ alors le feuilletage $\mathcal{G}$ est tangent «extérieurement» au feuilletage $\mathcal{F}$. On suppose que $\mathcal{G}$ est tangent «extérieurement» à $\mathcal{F}$ le long de cette demi-droite et on considère $G_{2}^{\prime}$ le morceau de feuille de $\mathcal{G}$ tangent au segment $F_{1} \cap F_{2}$. On note $D$ le disque ayant pour bord $G_{2}^{\prime} \cup G_{3}^{\prime}$ et le segment de droite fermant cette courbe. Les segments $G_{2}$ et $G_{3}$ sont à l'extérieur de $D$ alors que $F_{2}$ et $F_{3}$ sont à l'intérieur. On en déduit que $F_{3}$ et $G_{3}$ ne se rencontrent pas. On procède de même dans les demi-cônes suivants pour montrer l'affirmation.

Dans le premier cas de l'affirmation, le bigone formé par $F_{0}, F_{1}, G_{0}$ et $G_{1}$ vérifie la conclusion du lemme. Si l'un des coins du bigone est sur une droite de tangences, on peut adapter l'affirmation pour terminer la preuve du lemme.

Définition 4.2. Nous appellerons perle d'un couple de feuilletages $\mathcal{F}, \mathcal{G}$ de $\mathbb{R}^{2}$, tout bigone $\Delta$ tel que l'ensemble des points de tangence des feuilletages dans $\Delta$ privé de ses coins est un segment $\sigma$, topologiquement transverse aux feuilletages, et joignant un point $x \in \partial_{\mathcal{F}}(\Delta)$ à un point de $y \in \partial_{\mathcal{G}}(\Delta)$.

Le segment $\sigma$ orienté de $x$ vers $y$ s'appellera l'axe de la perle (voit Figure 3).

Toutes les perles sont identiques, à conjugaison près :

LEMme 4.3. Étant donné deux perles $\Delta_{1}, \Delta_{2}$ de deux couples de feuilletages $\left(\mathcal{F}_{1}, \mathcal{G}_{1}\right)$ et $\left(\mathcal{F}_{2}, \mathcal{G}_{2}\right)$, il existe un homéomorphisme préservant l'orientation $h: \Delta_{1} \rightarrow \Delta_{2}$ qui conjugue 
les restrictions à $\Delta_{1}$ des feuilletages $\mathcal{F}_{1}$ et $\mathcal{G}_{1}$ aux restrictions à $\Delta_{2}$ des feuilletages $\mathcal{F}_{2}$ et $\mathcal{G}_{2}$.

Nous allons définir une relation binaire sur l'ensemble des bigones d'un couple de feuilletages :

Définition 4.3. Soient $\Delta_{1}$ et $\Delta_{2}$ deux perles d'un couple de feuilletages de type radial. Nous dirons que $\Delta_{2}$ suit $\Delta_{1}$ si les trois conditions suivantes sont vérifiées :

$$
\begin{array}{ll}
- & \operatorname{int}\left(\Delta_{1}\right) \cap \operatorname{int}\left(\Delta_{2}\right) \neq \emptyset ; \\
- & \partial_{\mathcal{F}} \Delta_{1} \cap \Delta_{2}=\emptyset ; \\
- & \Delta_{1} \cap \partial_{\mathcal{G}} \Delta_{2}=\emptyset .
\end{array}
$$

On vérifie alors facilement :

Lemme 4.4. Si $\Delta_{1}$ et $\Delta_{2}$ sont deux perles telles que $\Delta_{2}$ suit $\Delta_{1}$, alors l'intersection $\Delta_{2} \cap \Delta_{1}$ est une perle $\Delta$ telle que $\partial_{\mathcal{F}} \Delta \subset \partial_{\mathcal{F}} \Delta_{2}$ et $\partial_{\mathcal{G}} \Delta \subset \partial_{\mathcal{G}} \Delta_{1}$.

Définition 4.4. Soit $I$ un intervalle de $\mathbb{Z}$. Nous dirons qu'une suite de perles $\left(\Delta_{n}\right)_{n \in I}$ est un chapelet de perles $\mathrm{si}$ on a :

- $\quad \Delta_{i+1}$ suit $\Delta_{i}$, pour tout $i \in \mathbb{N}$;

- $\quad \Delta_{i} \cap \Delta_{j}=\emptyset$ pour tout $i, j$ tels que $|i-j| \geq 2$.

COROLlaire 4.1. Soit $\left(\Delta_{n}\right)_{n \in I}$ un chapelet de perles d'un couple de feuilletages $(\mathcal{F}, \mathcal{G})$, et notons $\mathcal{D}=\bigcup_{n \in I} \Delta_{n}$ l'union des perles. Alors l'union $\Sigma$ des axes $\sigma_{i}$ des perles est une courbe plongée (compacte si l'intervalle I est fini) dont l'intersection avec chaque perle est exactement son axe. Elle coïncide avec l'ensemble des points de tangence dans $\mathcal{D}$ des feuilletages (privé éventuellement de certains coins des bigones).

Démonstration. On remarque que l'union $\mathcal{D}$ des perles de la suite est connexe. Soient $\Delta_{i}, \Delta_{i+1}$ deux perles successives du chapelet. L'intersection $\sigma_{i} \cap \sigma_{i+1}$ de leurs axes est l'axe de la perle $\Delta_{i} \cap \Delta_{i+1}$ et l'union $\sigma_{i} \cup \sigma_{i+1}$ est donc un segment plongé. Par définition de chapelet, $\sigma_{i} \cap \sigma_{j}=\emptyset$ pour $|i-j| \geq 2$, ce qui conclut.

On appellera fil du chapelet de perles $\left(\Delta_{i}\right)$ l'union des axes des perles. C'est une courbe de tangences entre les feuilletages, qui traverse successivement toutes les perles.

Remarque 4.5. Soit $i_{1}<i_{2}<\cdots<i_{k} \in I$ tels que $\left|i_{j}-i_{j+1}\right| \geq 2$ pour tout $j \in\{1, \ldots, k\}$. Choisissons un point $x_{i_{j}}$ sur chaque axe $\sigma_{i_{j}}$ de perles $\Delta_{i_{j}}$ du chapelet. Les points $\left\{x_{i_{j}}\right\}$ sont alors placés de façon monotone sur le fil $\Sigma$ du chapelet : si $j<m<n$ alors le point $x_{i_{m}}$ appartient à l'intérieur du segment $\left[x_{i_{j}}, x_{i_{n}}\right]$. Par convention on oriente le fil d'un chapelet de façon à ce que ces suites soient croissantes.

Les chapelets de perles vont nous servir à situer les lignes de tangences des feuilletages, comme étant le fil de ces chapelets. Nous allons à présent voir que l'existence d'un chapelet $\Delta_{i}$ pour un couple de feuilletage $(\mathcal{F}, \mathcal{G})$ permet de construire un chapelet pour un couple $\left(\mathcal{F}^{\prime}, \mathcal{G}^{\prime}\right)$, où $\mathcal{F}^{\prime}$ et $\mathcal{G}^{\prime}$ sont guidés par $\mathcal{F}$ et $\mathcal{G}$ respectivement, pourvu que l'on puisse réduire les perles $\Delta_{i}$. 
4.2. Les chapelets de perles et la relation $\approx$. Dans toute cette section $\left(\mathcal{F}=\mathcal{L}_{\alpha}\right.$, $\mathcal{G}=\mathcal{L}_{\beta}^{\lambda}$ ) est un couple de feuilletages logarithmiques présentant deux droites de tangences.

Le cercle unité est une transversale complète du feuilletage $\mathcal{F}$. La métrique naturelle sur le cercle unité munit donc l'espace des feuilles de $\mathcal{F}$ d'une distance. L'amplitude d'un secteur $S$ de $\mathcal{F}$ est alors la longueur du segment $J$ correspondant sur le cercle unité. Le secteur $S$ sera dit centré sur la feuille passant par $x$ si la feuille passant par $x$ correspond au centre de $J$

Le feuilletage $\mathcal{G}$ est l'image par la matrice $\left(\begin{array}{ll}1 & 0 \\ 0 & \lambda\end{array}\right)$ du feuilletage $\mathcal{L}_{\beta}$. L'amplitude d'un secteur de $\mathcal{G}$ sera par définition celle du secteur de $\mathcal{L}_{\beta}$ dont il est l'image.

Définition 4.5. On dira qu'un feuilletage $\mathcal{F}^{\prime}$ est $\varepsilon$-guidé par $\mathcal{F}$ sur un voisinage $U$ de l'origine si, pour tout point $x \in U$, le segment de feuille de $\mathcal{F}^{\prime}$ joignant $x$ à l'origine est inclus dans le secteur de $\mathcal{F}$ d'amplitude $\varepsilon$, centré sur la feuille de $\mathcal{F}$ passant par $x$.

On vérifie facilement qu'un feuilletage $\mathcal{F}^{\prime}$ est guidé par $\mathcal{F}$ (pour la Définition 2.1) si et seulement si, pour tout $\varepsilon>0$, il existe un voisinage de l'origine sur lequel $\mathcal{F}^{\prime}$ est $\varepsilon$-guidé $\operatorname{par} \mathcal{F}$.

Définition 4.6. Un $\mathcal{F}$-rectangle est un disque topologique inclus dans un secteur de $\mathcal{F}$, dont le bord est constitué de deux segments du bord de ce secteur et de deux segments topologiquement transverse à $\mathcal{F}$.

On appellera largeur d'un $\mathcal{F}$-rectangle l'amplitude du secteur sur lequel il s'appuie.

On définit de même les $\mathcal{G}$-rectangles ainsi que leur largeur.

Lemme 4.6. Soit $\mathcal{F}^{\prime}$ un feuilletage de type radial, soient $\varepsilon>0$ et $U$ un ouvert tel que $\mathcal{F}$ $\varepsilon$-guide $\mathcal{F}^{\prime}$ sur $U$. Alors, pour tout $\mathcal{F}$-rectangle $R \subset U$ de largeur $\varepsilon$, il existe un segment de feuille de $\mathcal{F}^{\prime}$, inclus dans $R$ et joignant les deux côtés transverses du rectangle.

Démonstration. Notons $S$ le secteur d'amplitude $\varepsilon$ sur lequel s'appuie le rectangle, et notons $L$ la feuille de $\mathcal{F}$ sur laquelle il est centré. On considère $\gamma_{0}$ le segment de bord transverse du rectangle, qui est le plus éloigné de l'origine dans le secteur, et on note $\gamma_{1}$ l'autre segment transverse. Soit $x=\gamma_{0} \cap L$. Par définition, le segment de feuille de $\mathcal{F}^{\prime}$ joignant $x$ à l'origine est inclus dans le secteur $S$. Notons $L_{x}^{\prime}$ ce segment. Soit $y \in L^{\prime}$ le dernier point d'intersection de $L^{\prime} \cap \gamma_{0}$. Le segment $L_{y}^{\prime} \subset L_{x}^{\prime}$ joignant $y$ à l'origine est bien sûr contenu dans le secteur $S$. Il doit donc couper le segment $\gamma_{1}$. Soit $z \in L_{y}^{\prime}$ le premier point d'intersection avec $\gamma_{1}$. Le segment de $L_{y}^{\prime}$ joignant $y$ à $z$ est le segment annoncé.

Soit $S$ un secteur de $\mathcal{F}$ et soit $\gamma \subset S$ un segment topologiquement transverse à $\mathcal{F}$ et joignant les deux composantes du bord de $S$. Le segment $\gamma$ coupe alors $S$ en deux demi-secteurs, qui seront désignés comme le demi-secteur vers l'origine noté $S_{\gamma}^{0}$, et le demi-secteur vers l'infini noté $S_{\gamma}^{\infty}$.

La même preuve que celle du Lemme 4.6 montre :

LEMme 4.7. Soit $\mathcal{F}^{\prime}$ un feuilletage E-guidé par $\mathcal{F}$ sur un ouvert $U$ et soit $S_{\gamma}^{0} \subset U$ un demi-secteur d'amplitude $\varepsilon$. Alors il existe un point y du segment $\gamma$ pour lequel le segment de feuille $L_{y}^{\prime}$ joignant y à l'origine est inclus dans $S_{\gamma}^{0}$. 
Définition 4.7. On appelle $\varepsilon$-perle pour les feuilletages $\mathcal{F}$ et $\mathcal{G}$ la donnée d'une perle $\Delta$, d'un $\mathcal{F}$-rectangle $R_{\mathcal{F}}(\Delta)$ de largeur $\varepsilon$, et d'un $\mathcal{G}$-rectangle $R_{\mathcal{G}}(\Delta)$ de largeur $\varepsilon$ tels que :

(1) $R_{\mathcal{F}}(\Delta)$ et $R_{\mathcal{G}}(\Delta)$ sont inclus dans $\Delta$;

(2) $R_{\mathcal{F}}(\Delta)$ admet $\partial_{\mathcal{F}}(\Delta)$ comme un de ses côtés tangents à $\mathcal{F}$, et ses côtés transverses à $\mathcal{F}$ sont inclus dans $\partial_{\mathcal{G}}(\Delta)$

(3) $R_{\mathcal{G}}(\Delta)$ admet $\partial_{\mathcal{G}}(\Delta)$ comme un de ses côtés tangents à $\mathcal{G}$, et ses côtés transverses à $\mathcal{G}$ sont inclus dans $\partial_{\mathcal{G}}(\Delta)$;

(4) $\quad \Delta \backslash\left(R_{\mathcal{F}}(\Delta) \cup R_{\mathcal{G}}(\Delta)\right.$ ) est non-vide (son adhérence est alors une perle notée $\Delta^{\epsilon}$ ).

Lemme 4.8. Soient $\mathcal{F}^{\prime}$ et $\mathcal{G}^{\prime}$ deux feuilletages de type radial. Soient $\varepsilon>0$ et $U$ un voisinage de 0 tels que, sur $U, \mathcal{F}^{\prime}$ soit $\varepsilon$-guidé par $\mathcal{F}$ et $\mathcal{G}^{\prime}$ soit $\varepsilon$-guidé par $\mathcal{G}$. Enfin, soit $\Delta \subset U$ une e-perle pour le couple $(\mathcal{F}, \mathcal{G})$.

Alors il existe un bigone $\Delta^{\prime}$ pour le couple $\left(\mathcal{F}^{\prime}, \mathcal{G}^{\prime}\right)$ tel que $\Delta^{\varepsilon} \subset \Delta^{\prime} \subset \Delta$.

Démonstration. D'après le Lemme 4.6 il existe un segment $\gamma_{0} \subset R_{\mathcal{F}}(\Delta)$ de feuille de $\mathcal{F}^{\prime}$ joignant les deux côtés transverses du rectangle $R_{\mathcal{F}}(\Delta)$. De même il existe un segment $\gamma_{1} \subset R_{\mathcal{G}}(\Delta)$ de feuille de $\mathcal{G}^{\prime}$, joignant les deux côtés transverses de $R_{\mathcal{G}}(\Delta)$.

Notons $R_{1}$ et $R_{2}$ les composantes connexes de $R_{\mathcal{F}}(\Delta) \cap R_{\mathcal{G}}(\Delta)$ : ce sont à la fois des $\mathcal{F}$-rectangles et de $\mathcal{G}$-rectangles. Remarquons que l'on a : $\gamma_{0} \cap \gamma_{1} \subset R_{1} \cup R_{2}$. On montre facilement que les segments $\gamma_{0}$ et $\gamma_{1}$ possèdent des points d'intersection dans $R_{1}$ et dans $R_{2}$.

Considérons l'ensemble des segments inclus dans $\gamma_{0}$, dont l'origine appartient à $R_{1} \cap \gamma_{0} \cap \gamma_{1}$ et dont l'extrémité appartient à $R_{2} \cap \gamma_{0} \cap \gamma_{1}$. On remarque que cet ensemble est inductif pour l'inclusion ce qui permet de choisir un tel segment $\gamma_{0}^{\prime}$ minimal pour l'inclusion. Remarquons que l'intérieur de ce segment est disjoint de $\gamma_{1}$. Notons $\gamma_{1}^{\prime}$ le segment de $\gamma_{1}$ joignant les extrémités de $\gamma_{0}^{\prime}$. La courbe $\gamma_{0}^{\prime} \cup \gamma_{1}^{\prime}$ est alors une courbe fermée simple bordant un disque $\Delta^{\prime}$ qui est un bigone du couple de feuilletages $\left(\mathcal{F}^{\prime}, \mathcal{G}^{\prime}\right)$.

Il reste à montrer que $\Delta^{\prime}$ contient la perle $\Delta^{\varepsilon}$. Pour cela remarquons d'abord que le bord de $\Delta^{\prime}$ est disjoint de l'intérieur de $\Delta^{\varepsilon}$ : la perle $\Delta^{\varepsilon}$ est alors soit disjointe de $\Delta^{\prime}$ soit incluse dans $\Delta^{\prime}$. Un segment traversant $R_{\mathcal{G}}(\Delta)$ entre $R_{1}$ et $R_{2}$ joint $\Delta^{\varepsilon}$ au complémentaire de $\Delta$ et donc aussi de $\Delta^{\prime}$, et son nombre d'intersection homologique avec $\partial\left(\Delta^{\prime}\right)$ est \pm 1 : en effet il est disjoint de $\gamma_{0}^{\prime}$ et son nombre d'intersection avec $\gamma_{1}^{\prime}$ est \pm 1 car $\gamma_{1}^{\prime}$ joint $R_{1}$ à $R_{2}$ dans $R_{\mathcal{G}}(\Delta)$.

Remarque 4.9. $\mathrm{Si}$, dans le Lemme 4.8, le couple $\left(\mathcal{F}^{\prime}, \mathcal{G}^{\prime}\right)$ était l'image par un homéomorphisme $h$ d'un couple $\left(\mathcal{L}_{\tilde{\alpha}}, \mathcal{L}_{\tilde{\beta}}^{\tilde{\tilde{\beta}}}\right)$ de feuilletages logarithmiques, alors tout bigone de $\left(\mathcal{F}^{\prime}, \mathcal{G}^{\prime}\right)$ serait une perle. Dans ce cas le Lemme 4.8 a donc montré l'existence d'une perle $\Delta^{\prime}$ du couple $\left(\mathcal{F}^{\prime}, \mathcal{G}^{\prime}\right)$ vérifiant $\Delta^{\varepsilon} \subset \Delta^{\prime} \subset \Delta$.

Définition 4.8. On dit qu'une $\varepsilon$-perle $\Delta_{2}$ suit une $\varepsilon$-perle $\Delta_{1}$ si :

(1) $R_{\mathcal{F}}\left(\Delta_{1}\right) \cap \Delta_{2}=\emptyset$ et $R_{\mathcal{G}}\left(\Delta_{2}\right) \cap \Delta_{1}=\emptyset$;

(2) $\operatorname{int}\left(\Delta_{1}^{\varepsilon}\right) \cap \operatorname{int}\left(\Delta_{2}^{\varepsilon}\right) \neq \varnothing$ (rappelons que $\Delta_{1}^{\varepsilon}$ et $\Delta_{2}^{\varepsilon}$ sont des perles contenues dans l'intérieur de $\Delta_{1}$ et $\Delta_{2}$, respectivement).

On dit qu'une suite $\Delta_{1}, \ldots, \Delta_{n}$ de $\varepsilon$-perles forme un chapelet de $\varepsilon$-perles si $\Delta_{i+1}$ suit $\Delta_{i}$ pour tout $i$, et si $\Delta_{i} \cap \Delta_{j}=\emptyset$ si $|i-j| \geq 2$. 
Corollaire 4.2. Soit $\left(\mathcal{F}^{\prime}, \mathcal{G}^{\prime}\right)$ un couple de feuilletages de type radial, tel que tout bigone de ce couple soit une perle. On suppose qu'il existe $\varepsilon>0$ et un voisinage $U$ de l'origine tel que $\mathcal{F}$ E-guide $\mathcal{F}^{\prime}$ et $\mathcal{G}$ E-guide $\mathcal{G}^{\prime}$ sur $U$. On suppose de plus que $\Delta_{1}, \ldots, \Delta_{n}, \ldots$ est un chapelet de e-perles incluses dans $U$, pour $(\mathcal{F}, \mathcal{G})$.

Alors il existe un chapelet de perles $\Delta_{i}^{\prime}$ pour le couple $\left(\mathcal{F}^{\prime}, \mathcal{G}^{\prime}\right)$, telles que $\Delta_{i}^{\varepsilon} \subset \Delta_{i}^{\prime} \subset \Delta_{i}$.

Démonstration. Le Lemme 4.8 assure l'existence, dans chaque perle $\Delta_{i}$, d'une perle $\Delta_{i}^{\prime}$ de $\left(\mathcal{F}^{\prime}, \mathcal{G}^{\prime}\right)$ contenant $\Delta_{i}^{\varepsilon}$. Par définition de chapelet de $\varepsilon$-perles, les intérieurs des perles $\Delta_{i}^{\varepsilon}$ et $\Delta_{i+1}^{\varepsilon}$ se rencontrent : il en est donc de même des intérieurs des perles $\Delta_{i}^{\prime}$ et $\Delta_{i+1}^{\prime}$. De plus $\partial_{\mathcal{F}^{\prime}} \Delta_{i}^{\prime} \subset R_{\mathcal{F}}\left(\Delta_{i}\right)$, donc est disjoint de $\Delta_{i+1}$ et a fortiori de $\Delta_{i+1}^{\prime}$. De même $\partial_{\mathcal{G}^{\prime}}\left(\Delta_{i+1}^{\prime}\right)$ est disjoint de $\Delta_{i}^{\prime}$, ce qui montre que $\Delta_{i}^{\prime}$ suit $\Delta_{i+1}^{\prime}$.

De plus pour tous $i, j$ tels que $|i-j| \geq 2$, les perles $\Delta_{i}$ et $\Delta_{j}$ sont disjointes, il en va donc de même des perles $\Delta_{i}^{\prime}$ et $\Delta_{j}^{\prime}$. On a montré que les $\Delta_{i}^{\prime}$ forment un chapelet.

4.3. Droites de tangences d'un couple $\left(h\left(\mathcal{L}_{\tilde{\alpha}}\right), h\left(\mathcal{L}_{\tilde{\beta}}^{\tilde{\lambda}}\right)\right)$ équivalent à $\left(\mathcal{L}_{\alpha}, \mathcal{L}_{\beta}^{\lambda}\right)$. Le paragraphe précédent a défini un objet, les chapelets de $\varepsilon$-perles, permettant de situer le lieu de tangence d'un couple de feuilletage $\left(\mathcal{F}^{\prime}, \mathcal{G}^{\prime}\right)$ équivalent à $\left(\mathcal{L}_{\alpha}, \mathcal{L}_{\beta}^{\lambda}\right)$. Pour nous en servir, il nous reste à montrer son existence. $C^{\prime}$ 'est ce que fait le lemme suivant.

On appelle secteur radial tout secteur du feuilletage radial.

LEMme 4.10. Soit $(\mathcal{F}, \mathcal{G})$ un couple de feuilletages logarithmiques ayant deux droites de tangences. Soit $\mathcal{C}$ un secteur radial contenant une demi-droite de tangences $D^{+}$dans son intérieur. Alors il existe $\varepsilon>0$ et un chapelet de $\varepsilon$-perles $\left(\Delta_{i}\right)_{i \in \mathbb{Z}}$ tels que :

(1) les perles sont incluses dans $\mathcal{C}$;

(2) il existe $v>0$ tel que $\Delta_{i+1}$ est l'image de $\Delta_{i}$ par l'homothétie de rapport $v$.

Démonstration. On vérifie facilement que, pour tout chapelet de perles invariant par une homothetie de rapport $0<v \neq 1$, il existe $\varepsilon>0$ tel que le chapelet soit un chapelet de $\varepsilon$-perles.

Si $\Delta_{0}$ est une perle incluse dans le secteur radial $\mathcal{C}$ et dont l'axe est un segment $I$ de $D^{+}$, choisissons $v>1$ tel que $I \cap v I$ soit un segment d'intérieur non vide, et que $I \cap v^{2} I=\emptyset$. Supposons que l'orientation de l'axe de $\Delta_{0}$ induise sur $D^{+}$l'orientation «fuyant l'origine». Alors $\Delta_{i}=v^{i} \Delta_{0}$ est une perle et la famille $\left(\Delta_{i}\right)_{i \in \mathbb{Z}}$ est un chapelet de perles.

Si l'orientation de l'axe est «vers l'origine», on choisit $\Delta_{i}=v^{-i} \Delta_{0}$.

Il reste à construire $\Delta_{0}$. En utilisant le fait que quand des feuilletages $\mathcal{L}_{\alpha}$ et $\mathcal{L}_{\beta}^{\lambda}$ possèdent deux droites de tangences, et que les tangences entre les feuilles sont quadratiques, on construit facilement une perle qui soit un voisinage arbitrairement petit d'un point de tangence donné, ce qui conclut.

La démonstration du Lemme 4.10 a mis en évidence deux types de perles : une perle sera dite positive ou négative, suivant que l'orientation de son axe est «fuyant l'origine $\gg$ ou «vers l'origine», respectivement. Nous dirons qu'une droite de tangences est positive ou négative suivant qu'elle est incluse dans un chapelet de perles positives ou négatives, 
respectivement. On se convainc facilement que tout couple de feuilletages logarithmiques ayant deux droites de tangences possède une droite positive et une droite négative.

Remarque 4.11. Dans le Lemme 4.10 on peut de plus imposer au rapport $v$ d'être arbitrairement proche de 1: il suffit pour cela, dans la preuve du lemme, de choisir la perle $\Delta_{0}$ suffisamment petite (ce qui implique que $\varepsilon$ soit, lui aussi, choisi petit).

Du Corollaire 4.2 et du Lemme 4.10 on déduit directement le corollaire suivant qui prouve le premier item de la Proposition 4.1 :

Corollaire 4.3. Soit $\left(\mathcal{L}_{\alpha}, \mathcal{L}_{\beta}^{\lambda}\right)$ un couple de feuilletages logarithmiques présentant deux droites de tangences $D^{+}$(positive) et $D^{-}$(négative). Soit $\left(\mathcal{L}_{\tilde{\alpha}}, \mathcal{L}_{\tilde{\beta}}^{\tilde{\lambda}}\right)$ un autre couple tel que $\left(\mathcal{L}_{\tilde{\alpha}}, \mathcal{L}_{\tilde{\beta}}^{\tilde{\lambda}}\right) \approx\left(\mathcal{L}_{\alpha}, \mathcal{L}_{\beta}^{\lambda}\right)$. Alors $\mathcal{L}_{\tilde{\alpha}}$ et $\mathcal{L}_{\tilde{\beta}}^{\tilde{\lambda}}$ possèdent deux droites de tangences $\tilde{D}^{+}$ (positive) et $\tilde{D}^{-}$(négative).

De plus, fixons un homéomorphisme $h$ de $\mathbb{R}^{2}$ tel que $h\left(\mathcal{L}_{\tilde{\alpha}}\right) \simeq \mathcal{L}_{\alpha}$ et $h\left(\mathcal{L}_{\tilde{\beta}}^{\tilde{\lambda}}\right) \simeq \mathcal{L}_{\beta}^{\lambda}$. Alors, pour tout secteur radial $\mathcal{C}$, contenant dans son intérieur une demi-droite $D_{i}^{ \pm} \subset D^{ \pm}$ de tangences du couple $\left(\mathcal{L}_{\alpha}, \mathcal{L}_{\beta}^{\lambda}\right)$, il existe une demi-droite de tangences $\tilde{D}_{i}^{ \pm} \subset \tilde{D}^{ \pm}$du couple $\left(\mathcal{L}_{\tilde{\alpha}}, \mathcal{L}_{\tilde{\beta}}^{\tilde{\lambda}}\right)$, et un voisinage $U$ de 0 tels que $h\left(\tilde{D}_{i}^{ \pm}\right) \cap U \subset \mathcal{C}$.

4.4. Invariance du signe de $\alpha$. Le but de cette partie est de montrer le lemme suivant, qui prouve l'item 2 de la Proposition 4.1.

Lemme 4.12. Soient $\left(\mathcal{L}_{\alpha}, \mathcal{L}_{\beta}^{\lambda}\right)$ et $\left(\mathcal{L}_{\tilde{\alpha}}, \mathcal{L}_{\tilde{\beta}}^{\tilde{\lambda}}\right)$ deux couples de feuilletages logarithmiques présentant chacun deux droites de tangences, et qui sont positivement similaires :

$$
\left(\mathcal{L}_{\alpha}, \mathcal{L}_{\beta}^{\lambda}\right) \approx_{+}\left(\mathcal{L}_{\tilde{\alpha}}, \mathcal{L}_{\tilde{\beta}}^{\tilde{\lambda}}\right)
$$

Alors $\alpha$ et $\tilde{\alpha}$ sont de même signe.

Démonstration. Fixons $\varepsilon \in] 0, \pi$ [ et un voisinage $U$ de l'origine sur lequel $\mathcal{L}_{\alpha} \varepsilon$-guide $h\left(\mathcal{L}_{\tilde{\alpha}}\right)$. Fixons $r_{0}>0$ tel que $U$ contienne le disque de centre $(0,0)$ de rayon $r_{0}$.

Considérons $D_{1}$ une demi-droite de tangences positive entre les feuilletages $\mathcal{L}_{\alpha}$ et $\mathcal{L}_{\beta}^{\lambda}$. Soit $V$ un secteur radial centré sur $D_{1}$ et disjoint des autres demi-droites de tangences. Pour tout $\left.r \in] 0, r_{0}\right]$, on notera $V_{r}$ l'intersection de ce secteur avec le disque de rayon $r$ centré en l'origine. On notera $C_{r}$ l'intersection de $V_{r}$ avec le cercle de rayon $r$.

D'après le Corollaire 4.3 , il existe une demi-droite $\tilde{D}_{1}$ de tangences des feuilletages $\left(\mathcal{L}_{\tilde{\alpha}}, \mathcal{L}_{\tilde{\beta}}^{\tilde{\lambda}}\right)$, telle que l'intersection de son image $D_{1}^{\prime}=h\left(\tilde{D}_{1}\right)$ avec un petit voisinage de $(0,0)$ soit incluse dans $V$. On en déduit que, pour $r<r_{0}$ assez petit, il existe un point $y_{r} \in C_{r} \cap D_{1}^{\prime}$ tel que le segment de $D_{1}^{\prime}$ joignant $y_{r}$ à l'origine soit inclus dans $V_{r}$.

On fixe un demi-secteur «vers l'origine» $S_{+}$de $\mathcal{L}_{\alpha}$, d'amplitude $\varepsilon$, inclus dans $U$, et dont le bord transverse est disjoint de $V$. D'après le Lemme 4.7, il existe un point $x$ du bord transverse de $S_{+}$tel que le segment $\gamma_{x}^{\prime}$ de feuille de $h\left(\mathcal{L}_{\tilde{\alpha}}\right)$ joignant $x$ à l'origine soit inclus dans $S_{+}$. On note $\gamma_{x}$ le segment de feuille de $\mathcal{L}_{\alpha}$ joignant $x$ à l'origine.

On remarque que chaque composante connexe de $V_{r} \cap S_{+}$est un $\mathcal{L}_{\alpha}$-rectangle d'amplitude $\varepsilon$ qui contient un point d'intersection de $\gamma_{x} \cap D_{1}$. Numérotons $x_{i}, i \in \mathbb{N}$, 
les points d'intersection de $\gamma_{x}$ avec $D_{1}$, cette numérotation étant faite vers l'origine $(0,0)$ (le long de $\gamma_{x}$ ou de $D_{1}$, ces intersections se faisant de façon monotone), et on note $R_{i}$ la composante de $V_{r} \cap S_{+}$contenant $x_{i}$.

Nous laissons au lecteur le soin de montrer les deux lemmes suivants.

Lemme 4.13. Pour tout $i \in \mathbb{N}$, le $\mathcal{L}_{\alpha}$-rectangle $R_{i}$ contient un et un seul point $x_{i}^{\prime} \in$ $\gamma_{x}^{\prime} \cap D_{1}^{\prime}$.

Lemme 4.14. Les points $x_{i}^{\prime}$ sont ordonnés sur $\gamma_{x}^{\prime}$ et sur $D_{1}^{\prime}$ de façon monotone, vers l'origine $(0,0)$.

Notons $H$ l'application de premier retour, sur la demi-droite $D_{1}$, du feuilletage $\mathcal{L}_{\alpha}$ parcouru en sens trigonométrique direct. L'application $H$ est l'homothétie de rapport $e^{2 \pi / \alpha}$. C'est une contraction si $\alpha<0$ et une dilatation sinon. On en déduit que $x_{i}=H^{i}\left(x_{0}\right)$ si $\alpha<0$ et $x_{i}=H^{-i}\left(x_{0}\right)$ si $\alpha>0$.

On considère alors le lacet $a_{n}$ (resp. $a_{n}^{\prime}$ ) formé des segments de $\gamma_{x}$ et de $D_{1}$ (resp. $\gamma_{x}^{\prime}$ et $D_{1}^{\prime}$ ) joignant $x_{0}$ à $x_{n}$ (resp. $x_{0}^{\prime}$ à $x_{n}^{\prime}$ ). On oriente ces lacets de façon que les segments sur $\gamma_{x}$ et $\gamma_{x}^{\prime}$ soient orientés de $x_{0}$ vers $x_{n}$ et de $x_{0}^{\prime}$ vers $x_{n}^{\prime}$, respectivement.

On vérifie que les lacets $a_{n}$ et $a_{n}^{\prime}$ ainsi orientés, sont librement homotopes dans $\mathbb{R}^{2} \backslash\{(0,0)\}$.

Remarquons que $\pi_{1}\left(\mathbb{R}^{2} \backslash\{(0,0)\}\right)=\mathbb{Z}$, le générateur 1 correspondant à un cercle orienté dans le sens trigonométrique. La classe d'homotopie de $a_{n}$ est alors $+n$ si $\alpha<0$ (en effet $x_{n}=H^{n}\left(x_{0}\right)$ ) et est $-n$ sinon.

On considère $\tilde{a}_{n}=h^{-1}\left(a_{n}^{\prime}\right)$ et $\tilde{x}_{i}=h^{-1}\left(x_{i}^{\prime}\right)$. Comme $h$ préserve l'orientation, il induit l'identité sur $\pi_{1}\left(\mathbb{R}^{2} \backslash\{(0,0)\}\right)$. On en déduit que $\tilde{a}_{n}$ représente le même élément que $a_{n}^{\prime}$ (et donc que $\left.a_{n}\right)$ dans $\pi_{1}\left(\mathbb{R}^{2} \backslash\{(0,0)\}\right)=\mathbb{Z}$. D'autre part $\tilde{a}_{n}$ est formé d'un segment de feuille de $\mathcal{L}_{\tilde{\alpha}}$ et d'un segment de $\tilde{D}_{1}$. Notons $\tilde{H}$ l'holonomie de $\mathcal{L}_{\tilde{\alpha}}$ (orienté dans le sens trigonométrique direct) sur $\tilde{D}_{1}$. On en déduit que $\tilde{x}_{n}=\tilde{H}^{n}\left(\tilde{x}_{0}\right)$ si $\alpha<0$ et $\tilde{x}_{n}=\tilde{H}^{-n}\left(\tilde{x}_{0}\right)$ si $\alpha>0$. D'autre part, d'après le Lemme $4.14, \tilde{x}_{n}$ est plus proche de $(0,0)$ sur $\tilde{D}_{1}$ que $\tilde{x}_{0}$.

On vient de voir que $H$ et $\tilde{H}$ sont simultanément des contractions ou des dilatations, ce qui revient à dire que $\alpha$ et $\tilde{\alpha}$ sont de même signe.

4.5. Invariance $d u$ rapport $\alpha / \beta$. Le but de cette section est de prouver l'item 3 de la Proposition 4.1.

Le rapport $\alpha / \beta$ a un sens dynamique pour le couple $\left(\mathcal{L}_{\alpha}, \mathcal{L}_{\beta}^{\lambda}\right)$ : c'est le nombre de translation relatif des applications de premier retour de ces feuilletages sur une demidroite quelconque, en particulier les demi-droites de tangences. Les droites de tangences étant préservées par conjugaison (i.e. équivalence topologique), ce rapport est un invariant d'équivalence topologique du couple de feuilletages. Toute la difficulté provient ici de ce que la similarité est une relation beaucoup plus faible que l'équivalence topologique.

Rappelons d'abord la définition du nombre de translation relatifs de deux homéomorphismes commutants de $\mathbb{R}$.

4.5.1. Nombre de translation relatif. Soient $f$ et $g$ deux homéomorphismes de $\mathbb{R}$, qui commutent : $f \circ g(x)=g \circ f(x)$ pour tout $x$ de $\mathbb{R}$. On suppose de plus que $f$ n'a pas de 
point fixe. Soit $x$ un point de $\mathbb{R}$ et $n$ un élément de $\mathbb{N}$, on note $m(n)$ l'entier vérifiant :

$$
f^{m(n)}(x) \leq g^{n}(x)<f^{m(n) \pm 1}(x) .
$$

Lemme et Définition 4.15. Pour tout $x \in \mathbb{R}$ la limite $\lim _{n \rightarrow \pm \infty} m(n) / n$ existe, de plus elle ne dépend pas de $x$. On la note $\rho(g ; f)$ et on l'appelle le nombre de translation relatif de $g$ par rapport à $f$.

\section{Remarque 4.16.}

(1) Comme $f$ est sans point fixe, le quotient de $\mathbb{R}$ par $f$ (autrement dit l'espace des orbites de $f$ ) est homéomorphe au cercle $S^{1}$. Comme $g$ commute avec $f$, il induit, par passage au quotient sur $S^{1}$, un homéomorphisme du cercle noté $g / f$. Le nombre de rotation (de Poincaré) de l'homéomorphisme $g / f$ est la classe modulo $\mathbb{Z}$ de $\rho(g ; f)$.

(2) Le nombre $\rho(g ; f)$ est invariant par conjugaison topologique. Ceci permet de généraliser la définition du nombre de translation relatif au cas d'homéomorphismes commutants $f$ et $g$ d'un intervalle (ou d'une courbe) pourvu que $f$ n'ait pas de point fixe sur cet intervalle.

(3) Pour tout $n, m \in \mathbb{Z}$, le produit $g^{n} \circ f^{m}$ est un homéomorphisme commutant avec $f$, et $\rho\left(g^{n} \circ f^{m} ; f\right)=n \rho(g ; f)+m$.

(4) Le nombre de translation relatif $\rho(g ; f)$ est positif ou nul si et seulement s'il existe un point $x$ tel que $(g(x)-x)(f(x)-x) \geq 0$, et il est nul si et seulement si $g$ possède un point fixe.

Les propriétés ci-dessus sont classiques, et les preuves sont identiques à celles existantes pour les nombres de rotation d'homéomorphismes du cercle. Signalons cependant [3, §3.b] où toutes ces propriétés sont démontrées sous des hypothèses plus faibles ( $f$ et $g$ sont des homéomorphismes locaux).

On peut calculer $\rho(g ; f)$ de la façon suivante :

Lemme 4.17. Soient $x, y$ deux points de $\mathbb{R}$ et $m, n, r, s, t \in \mathbb{Z}$, tels que $f^{m}(x) \leq y \leq$ $f^{n}(x)$ et $f^{r}(x) \leq g^{s}(y) \leq f^{t}(x)$. Alors,

$$
\inf \left\{\frac{t-m}{s}, \frac{r-n}{s}\right\} \leq \rho(g ; f) \leq \sup \left\{\frac{t-m}{s}, \frac{r-n}{s}\right\} \text {. }
$$

Démonstration. En effet $f^{r-n}(y) \leq g^{s}(y) \leq f^{t-m}(y)$. Il suffit alors d'utiliser les items 3 et 4 de la Remarque 4.16 ci-dessus.

Remarque 4.18. Si $f$ et $g$ sont des homothéties de $\mathbb{R}$ de rapports positifs $\alpha_{f}$ et $\alpha_{g}$, le nombre de translation relatif des restrictions de $f$ et $g$ à $] 0,+\infty[$ (ou à $]-\infty, 0[$ ) est

$$
\rho(g ; f)=\frac{\log \left(\alpha_{g}\right)}{\log \left(\alpha_{f}\right)} .
$$

En effet, les conjuguées des homothéties $g$ et $f$ par l'application log: $] 0,+\infty[\rightarrow \mathbb{R}$ sont les translations de pas $\log \left(\alpha_{g}\right)$ et $\log \left(\alpha_{f}\right)$, respectivement. 
4.5.2. Application aux feuilletages logarithmiques. Soit $D$ une demi-droite vectorielle de $\mathbb{R}^{2}$, et soit $\left(\mathcal{L}_{\alpha}, \mathcal{L}_{\beta}^{\lambda}\right)$ un couple de feuilletages logarithmiques sur $D$. Les applications de premier retour $H_{\alpha, D}$ et $H_{\beta, \lambda, D}$ de ces feuilletages (orientés dans le sens direct) sur $D$ sont les homothéties de rapport $e^{2 \pi / \alpha}$ et $e^{2 \pi / \beta}$ respectivement, si bien que $\alpha / \beta$ est le nombre de translation relatif de $H_{\beta, \lambda, D}$ par rapport à $H_{\alpha, D}$.

La proposition suivante rappelle l'item 3 de la Proposition 4.1 :

Proposition 4.2. Soient $\left(\mathcal{L}_{\alpha}, \mathcal{L}_{\beta}^{\lambda}\right) \approx_{+}\left(\mathcal{L}_{\tilde{\alpha}}, \mathcal{L}_{\tilde{\beta}}^{\tilde{\lambda}}\right)$ deux couples de feuilletages positivement similaires, ayant chacun deux droites de tangences. Alors :

$$
\frac{\alpha}{\beta}=\frac{\tilde{\alpha}}{\tilde{\beta}} .
$$

La démonstration de cette proposition est l'objet de toute cette section.

Afin de simplifier les notations on notera $\mathcal{F}=\mathcal{L}_{\alpha}, \mathcal{G}=\mathcal{L}_{\beta}^{\lambda}, \tilde{\mathcal{F}}=\mathcal{L}_{\tilde{\alpha}}$ et $\tilde{\mathcal{G}}=\mathcal{L}_{\tilde{\beta}}^{\tilde{\lambda}}$. Par hypothèse, il existe un homéomorphisme $h$ préservant l'orientation tel que $\mathcal{F} \simeq \mathcal{F}^{\prime}=$ $h(\tilde{\mathcal{F}})$ et $\mathcal{G} \simeq \mathcal{G}^{\prime}=h(\tilde{\mathcal{G}})$.

D'après le Lemme $4.12, \alpha$ et $\tilde{\alpha}$ sont de même signe. On supposera donc, sans perte de généralité, que $\alpha$ et $\tilde{\alpha}$ sont négatifs : les applications de retour de ces feuilletages, parcourus dans le sens trigonométrique direct, sur des demi-droites vectorielles sont donc des contractions. Remarquons que le Lemme 4.12 implique directement que $\beta$ et $\tilde{\beta}$ sont également de même signe.

On considère une demi-droite de tangences $D$ de $\mathcal{F}$ avec $\mathcal{G}$. On sait d'après le Corollaire 4.3 qu'il existe une demi-droite de tangences $\tilde{D}$ de $\tilde{\mathcal{F}}$ avec $\tilde{\mathcal{G}}$ dont l'image $D^{\prime}=h(\tilde{D})$ est incluse près de l'origine dans des secteurs radiaux arbitrairement fins autour de $D$.

On note $f$ et $g$ les applications de retour sur $D$ des feuilletages $\mathcal{F}$ et $\mathcal{G}$, respectivement, parcourus en sens trigonométrique. Bien que les feuilletages $\mathcal{F}^{\prime}$ et $\mathcal{G}^{\prime}$ et la courbe $D^{\prime}$ ne soient à priori pas différentiables, les applications de premier retour sur $D^{\prime}$ de ces feuilletages «parcourus dans le sens direct» sont bien définies : ce sont les conjuguées par $h$ des applications de premier retour $\tilde{f}$ et $\tilde{g}$ de $\tilde{\mathcal{F}}$ et $\tilde{\mathcal{G}}$ sur $\tilde{D}$. En particulier, $f^{\prime}$ et $g^{\prime}$ commutent et leur nombre de translation relatif $\rho\left(g^{\prime}, f^{\prime}\right)$ est $\tilde{\alpha} / \tilde{\beta}$.

La Proposition 4.2 est un corollaire direct du lemme :

Lemme 4.19. Il existe $x, y \in D^{\prime}$ tels que pour tout $r, s, t \in \mathbb{Z}$, vérifiant $r / t \leq \alpha / \beta \leq s / t$, et pour tout $N>0$, il existe $k \in \mathbb{Z},|k|>N$, tel que $\left(g^{\prime}\right)^{k t}(y)$ appartienne au segment $\left[\left(f^{\prime}\right)^{k r}(x),\left(f^{\prime}\right)^{k s}(x)\right]_{D^{\prime}}$ de $D^{\prime}$ joignant les points $\left(f^{\prime}\right)^{k r}(x)$ et $\left(f^{\prime}\right)^{k s}(x)$.

Déduisons d'abord de ce lemme la Proposition 4.2.

Démonstration de la Proposition 4.2. On fixe $n_{0}$ tel que $y \in\left[\left(f^{\prime}\right)^{n_{0}}(x),\left(f^{\prime}\right)^{n_{0}+1}(x)\right]_{D^{\prime}}$. D'après le Lemme 4.17, le nombre de translation relatif de $g^{\prime}$ par rapport à $f^{\prime}$ appartient à un intervalle du type $\left[\left(k r \pm n_{0}\right) / k t,\left(k s \pm n_{0}\right) / k t\right]$. Il suffit alors de choisir $r, s, t$ de façon que cet intervalle soit arbitrairement petit, et le nombre de translation de $g^{\prime}$ par rapport à $f^{\prime}$ est donc $\alpha / \beta$, ce qui conclut.

Il reste donc à montrer le Lemme 4.19. 
Démonstration. Nous allons essayer de présenter toutes les idées de la preuve en évitant d'être inutilement technique.

On choisit $\varepsilon>0,0<v<1$, un secteur radial $V$ centré sur $D^{\prime}$, un voisinage ouvert $U$ de l'origine et un disque $D_{\rho}$ centré en l'origine tel que :

- le secteur $V$ contient un chapelet de $\varepsilon$-perles $\Delta_{i}$, invariant par l'homothetie de rapport $v$;

- $\quad$ les feuilletages $\mathcal{F}^{\prime}$ et $\mathcal{G}^{\prime}$ sont $\varepsilon$-guidés par $\mathcal{F}$ et $\mathcal{G} \operatorname{sur} U$;

- $\quad$ le disque $D_{\rho}$ est inclus dans $U$;

- $\quad$ notons $V_{\rho}=V \cap D_{\rho}$, et $C_{\rho}=V_{\rho} \cap \partial D_{\rho}$; il existe un point $z \in C_{\rho}$ tel que le segment $D_{z}^{\prime}$ de $D^{\prime}$ joignant $z$ à l'origine soit inclus dans $V_{\rho}$.

On choisit alors un point $x$ de $D_{\rho} \backslash V$ et un demi-secteur $S_{\mathcal{F}}$ vers l'origine du feuilletage $\mathcal{F}$, d'amplitude $\varepsilon$, centré en la feuille de $\mathcal{F}$ passant par $x$, de façon que $S_{\mathcal{F}} \subset D_{\rho}$ et que le bord transverse de $S_{\mathcal{F}}$ soit disjoint de $V$. Le Lemme 4.7 assure l'existence d'un point $x^{\prime}$ du bord transverse de $S_{\mathcal{F}}$ dont le segment $\mathcal{F}_{x^{\prime}}^{\prime}$ de feuille de $\mathcal{F}^{\prime}$ joignant $x^{\prime}$ à l'origine soit inclus dans $S_{\mathcal{F}}$. On note $\mathcal{F}_{x}$ le segment de feuille de $\mathcal{F}$ joignant $x$ à l'origine.

On note $R_{i}, i \in \mathbb{N}$, les composantes connexes de $S_{\mathcal{F}} \cap V_{\rho}$, numérotées de façon croissante, de $x$ vers l'origine. Chaque composante $R_{i}$ contient exactement un point $x_{i}$ de $\mathcal{F}_{x} \cap D$ et $R_{i}$ contient exactement un point $x_{i}^{\prime}$ de $\mathcal{F}_{x^{\prime}}^{\prime} \cap D_{z}^{\prime}$. De plus, en utilisant $\alpha<0$ et $\tilde{\alpha}<0$, on montre que $x_{i}=f^{i}\left(x_{0}\right)$ et $x_{i}^{\prime}=\left(f^{\prime}\right)^{i}\left(x_{0}^{\prime}\right)$.

On fait la même construction pour les feuilletages $\mathcal{G}$ et $\mathcal{G}^{\prime}$ : on choisit un point $y$ de $D_{\rho} \backslash V$ et demi-secteur $S_{\mathcal{G}}$ du feuilletage $\mathcal{G}$, d'amplitude $\varepsilon$, centré en la feuille de $\mathcal{G}$ passant par $y$, de façon que $S_{\mathcal{G}} \subset D_{\rho}$ et que le bord transverse de $S_{\mathcal{G}}$ soit disjoint de $V$. Il existe alors un point $y^{\prime}$ du bord transverse de $S_{\mathcal{G}}$ dont le segment $\mathcal{G}_{y^{\prime}}^{\prime}$ de feuille de $\mathcal{G}^{\prime}$ joignant $y^{\prime}$ à l'origine soit inclus dans $S_{\mathcal{G}}$. On note $\mathcal{G}_{y}$ le segment de feuille de $\mathcal{G}$ joignant $y$ à l'origine.

On note $S_{i}, i \in \mathbb{N}$ les composantes connexes de $S_{\mathcal{G}} \cap V_{\rho}$, numérotées de façon croissante de $y$ vers l'origine. Chaque composante $S_{i}$ contient exactement un point $y_{i}$ de $\mathcal{G}_{y} \cap D$ et un point $y_{i}^{\prime}$ de $\mathcal{G}_{y^{\prime}}^{\prime} \cap D_{z}^{\prime}$. De plus, si $\iota \in\{-1,1\}$ est l'inverse du signe de $\beta$ (et donc aussi de $\tilde{\beta})$ alors $y_{i}=f^{i i}\left(y_{0}\right)$ et $y_{i}^{\prime}=\left(g^{\prime}\right)^{i i}\left(y_{0}^{\prime}\right)$.

Par définition du nombre de translation relatif de $g$ par rapport à $f$ et par choix de $r, s, t$, pour tout $k$ assez grand on a :

$$
y_{l k t} \text { appartient au segment }\left[x_{k r}, x_{k s}\right]_{D} \text { de } D \text {. }
$$

Il suffit à présent de montrer que les points $y_{t k t}^{\prime}, x_{k r}^{\prime}$ et $x_{k s}^{\prime}$ sont ordonnés sur $D^{\prime}$ comme $y_{l k t}, x_{k r}$ et $x_{k s}$ sur $D$. Pour cela, en utilisant le fait que $\left(\Delta_{i}\right)$ est un chapelet de $\varepsilon$-perles et que les secteurs $S_{\mathcal{F}}$ et $S_{\mathcal{G}}$ sont d'amplitude $\varepsilon$, on vérifie facilement :

Lemme 4.20. Pour chacune des composantes $R_{i}$ ou $S_{i}$, l'intersection avec l'union des perles $\mathcal{D}=\bigcup_{i} \Delta_{i}$ est incluse dans deux perles successives.

Du Lemme 4.20, on déduit que, pour tout $i$, les points $x_{i}$ et $x_{i}^{\prime}$ sont soit dans la même perle, soit dans deux perles successives, et il en va de même pour des points $y_{i}$ et $y_{i}^{\prime}$. Rappelons que, par définition d'un chapelet, les perles $\Delta_{i}$ et $\Delta_{j}$ sont disjointes dès que $|i-j| \geq 2$. D'après la Remarque 4.5 , si trois points de $D^{\prime}$ appartiennent à des perles dont les indices diffèrent de plus de 2, alors leur ordre respectif sur $D^{\prime}$ est donné par celui des indices des perles. On conclut alors par le lemme suivant : 
LEMME 4.21. Pour $k$ assez grand les points $y_{t k t}, x_{k r}$ et $x_{k s}$ appartiennent à des perles $\Delta_{i}$, $\Delta_{j}$ et $\Delta_{k}$ avec $|i-j| \geq 5,|i-k| \geq 5$ et $|j-k| \geq 5$.

Démonstration. En effet, par choix du chapelet de $\varepsilon$-perles $\Delta_{i}$, deux perles successives sont multiples l'une de l'autre par $v \in] 0,1\left[\right.$. On en déduit que, si deux points $z_{1}, z_{2}$ de $D$ vérifient que $z_{2}$ est plus proche de l'origine que $v^{5} \cdot z_{1}$ (ce que l'on notera $z_{2} / z_{1} \leq v^{5}$ ) alors $z_{1}$ et $z_{2}$ appartiennent à des perles $\Delta_{i_{1}}$ et $\Delta_{i_{2}}$ avec $i_{2}-i_{1} \geq 5$.

L'expression explicite de $f$ et de $g$ (homothéties de rapport respectif $e^{2 \pi / \alpha}$ et $e^{2 \pi / \beta}$ ) permet de montrer que, quand $k$ tend vers l'infini, les rapports $x_{k s} / y_{k t}$ et $y_{k t} / x_{k r}$ tendent vers 0 , si $0<r<s$ (dans ce cas $k>0$ ) ou si $s<r<0$ (dans ce cas $k<0$ ), et vers $+\infty$ si $0<s<r$ (dans ce cas $k>0$ ) ou si $r<s<0$ (dans ce cas $k<0$ ). Ces rapports sont soit plus petits que $v^{5}$, soit plus grands que $v^{-5}$, ce qui conclut.

On en déduit que les points $y_{t k t}^{\prime}, x_{k r}^{\prime}$ et $x_{k s}^{\prime}$ sont ordonnés sur $D^{\prime}$ de la même façon que $y_{t k t}, x_{k r}$ et $x_{k s}$ sur $D$, ce qui conclut la preuve du Lemme 4.19 .

4.6. Invariance du nombre $\Psi(\alpha, \beta, t)$. On considère des feuilletages $\mathcal{F}=\mathcal{L}_{\alpha}, \mathcal{G}=\mathcal{L}_{\beta}^{\lambda}$, $\tilde{\mathcal{F}}=\mathcal{L}_{\tilde{\alpha}}$ et $\tilde{\mathcal{G}}=\mathcal{L}_{\tilde{\beta}}^{\tilde{\lambda}}$, tels que $\mathcal{F}$ et $\mathcal{G}$ sont tangents le long de deux droites $D_{+}$(droite de tangences positive) et $D_{-}$(droite de tangences négative), et tels que $(\mathcal{F}, \mathcal{G}) \approx_{+}(\tilde{\mathcal{F}}, \tilde{\mathcal{G}})$. On fixe donc un homéomorphisme $h$ de $\mathbb{R}^{2}$, préservant l'orientation, tel que $\mathcal{F} \simeq \mathcal{F}^{\prime}=$ $h(\tilde{\mathcal{F}})$ et $\mathcal{G} \simeq \mathcal{G}^{\prime}=h(\tilde{\mathcal{G}})$. Comme précédemment on notera $t=\frac{1}{2}(\lambda+1 / \lambda)$ et $\tilde{t}=\frac{1}{2}(\tilde{\lambda}+1 / \tilde{\lambda})$.

On rappelle que l'on a montré que le couple $(\tilde{\mathcal{F}}, \tilde{\mathcal{G}})$ possède aussi deux droites de tangences $\tilde{D}_{+}$et $\tilde{D}_{-}$(respectivement positive et négative), que $\alpha$ et $\tilde{\alpha}$ sont de même signe et que $\alpha / \beta=\tilde{\alpha} / \tilde{\beta}$.

Rappelons que dans la $\S 3.3$, on a choisi un cône, noté $S_{0}$, bordé par les droites $D_{+}$ et $D_{-}$et tel que sur ce cône l'orientation des vecteurs $X_{\alpha}$ et $X_{\beta}^{\lambda}$ soit opposée au signe de $\beta-\alpha$. On vérifie que, selon le signe de $\beta-\alpha, D_{-}$est l'origine ou l'extrémité des segments traversant $S_{0}$ dans le sens trigonométrique direct. Afin de fixer les idées on supposera que $D_{-}$est l'origine de ces segments.

On choisit de même le cône $\tilde{S}_{0}$ bordé par les droites $\tilde{D}_{+}$et $\tilde{D}_{-}$. Remarquons que, comme $\tilde{\alpha} / \tilde{\beta}=\alpha / \beta$ et que $\alpha$ et $\tilde{\alpha}$ sont de même signe, les signes de $\beta-\alpha$ et de $\tilde{\beta}-\tilde{\alpha}$ sont identiques. En conséquence $\tilde{D}_{-}$est l'origine des segments traversant $\tilde{S}_{0}$ dans le sens trigonométrique direct.

On note $H_{1}$ et $H_{2}$ les holonomies de $D_{-}$sur $D_{+}$des restrictions à $S_{0}$ de $\mathcal{F}$ et $\mathcal{G}$, respectivement. On note $H=H_{2}^{-1} \circ H_{1}$. On note $f$ l'application de deuxième retour sur $D_{-}$de $\mathcal{F}$, parcouru dans le sens direct (le choix du deuxième retour revient à considérer le premier retour sur une demi-droite). Rappelons que $f$ et $H$ sont des homothéties de $D_{-}$de rapports positifs, respectivement $e^{2 \pi / \alpha}$ et $\mu(\alpha, \beta, t)$. Par définition de la fonction $\Psi$ (voir la §3.3), $\Psi(\alpha, \beta, t)$ est égal à $(\alpha / 2 \pi) \log (\mu(\alpha, \beta, t))$. D'après la Remarque 4.18, $\Psi(\alpha, \beta, t)$ est le nombre de translation relatif $\rho(H ; f)$ de $H$ relativement à $f$.

On définit de même $\tilde{H}_{1}, \tilde{H}_{2}, \tilde{H}=\tilde{H}_{2}^{-1} \circ \tilde{H}_{1}$ et $\tilde{f}$. Alors $\tilde{f}$ et $\tilde{H}$ sont des homothéties de $\tilde{D}_{-}$de rapports respectifs $e^{2 \pi / \tilde{\alpha}}$ et $\mu(\tilde{\alpha}, \tilde{\beta}, \tilde{t})$ et $\Psi(\tilde{\alpha}, \tilde{\beta}, \tilde{t})=(\tilde{\alpha} / 2 \pi) \log (\mu(\tilde{\alpha}, \tilde{\beta}, \tilde{t}))$ est le nombre de translation relatif $\rho(\tilde{H} ; \tilde{f})$. 
On note $\mathcal{F}^{\prime}$ et $\mathcal{G}^{\prime}$ les images par $h$ des feuilletages $\tilde{\mathcal{F}}$ et $\tilde{\mathcal{G}}$. On note $D_{+}^{\prime}$ et $D_{-}^{\prime}$ les images par $h$ des droites $\tilde{D}_{+}$et $\tilde{D}_{-}$. Les courbes $D_{+}^{\prime}$ et $D_{-}^{\prime}$ sont les points de tangence topologique des feuilletages $\mathcal{F}^{\prime}$ et $\mathcal{G}^{\prime}$. On notera $H_{1}^{\prime}: D_{-}^{\prime} \rightarrow D_{+}^{\prime}, H_{2}^{\prime}: D_{-}^{\prime} \rightarrow D_{+}^{\prime}, H^{\prime}: D_{-}^{\prime} \rightarrow D_{-}^{\prime}$, $f^{\prime}: D_{-}^{\prime} \rightarrow D_{-}^{\prime}$ les conjugués par l'homéomorphisme $h$ des applications $\tilde{H}_{1}, \tilde{H}_{2}, \tilde{H}$ et $\tilde{f}$. Remarquons que $\rho\left(H^{\prime} ; f^{\prime}\right)=\rho(\tilde{H} ; \tilde{f})=\Psi(\tilde{\alpha}, \tilde{\beta}, \tilde{t})$.

Le Corollaire 4.3 montre que $D_{+}^{\prime}$ est contenue dans le cône $C_{+}^{\eta}$, d'angle $\eta>0$ arbitrairement petit, centré sur la droite $D_{+}$et de même $D_{-}^{\prime}$ est contenue dans le cône $C_{-}^{\eta}$, d'angle $\eta$, centré sur la droite $D_{-}$.

On définit enfin $S_{0}^{\prime}=h\left(\tilde{S}_{0}\right)$. Les applications $H_{1}^{\prime}, H_{2}^{\prime}$ sont les holonomies, de $D_{-}^{\prime}$ vers $D_{+}^{\prime}$ des restrictions à $S_{0}^{\prime}$ des feuilletages $\mathcal{F}^{\prime}$ et $\mathcal{G}^{\prime}$. Le lemme suivant exprime que $S_{0}^{\prime}$ est proche (au voisinage de $(0,0)$ ) du cône $S_{0}$.

Lemme 4.22. Pour tout $\eta>0$, il existe $\rho>0$ tel que l'image $S_{0}^{\prime}=h\left(\tilde{S}_{0}\right)$ contient l'intersection $D_{\rho} \cap\left(S_{0} \backslash\left(C_{+}^{\eta} \cup C_{-}^{\eta}\right)\right)$ où $D_{\rho}$ est le disque centré en $(0,0)$ de rayon $\rho$. De plus, $S_{0}^{\prime} \cap D_{\rho}$ est inclus dans $S_{0} \cup\left(C_{+}^{\eta} \cup C_{-}^{\eta}\right)$.

Démonstration. On choisit $\rho_{0}$ de sorte que $D_{\rho_{0}} \cap\left(D_{+}^{\prime} \cup D_{-}^{\prime}\right)$ soit inclus dans $C_{+}^{\eta} \cup C_{-}^{\eta}$. En conséquence, les feuilletages $\mathcal{F}^{\prime}$ et $\mathcal{G}^{\prime}$ sont topologiquement transverses sur $D_{\rho_{0}} \cap\left(S_{0} \backslash\right.$ $\left.\left(C_{+}^{\eta} \cup C_{-}^{\eta}\right)\right)$. On choisit $\varepsilon>0$ tel qu'il existe un $\mathcal{F}$-rectangle $R$, de largeur $\varepsilon$, qui soit également un $\mathcal{G}$-rectangle de largeur $\varepsilon$, inclus dans $D_{\rho_{0}} \cap\left(S_{0} \backslash\left(C_{+}^{\eta} \cup C_{-}^{\eta}\right)\right)$. On choisit alors $\rho<\rho_{0}$ tel que $\mathcal{F}$ et $\mathcal{G} \varepsilon$-guident $\mathcal{F}^{\prime}$ et $\mathcal{G}^{\prime}$ (respectivement) sur $D_{\rho}$. Par invariance des feuilletages $\mathcal{F}$ et $\mathcal{G}$ par homothétie, on peut supposer que $R$ est inclus dans $D_{\rho}$.

Le Lemme 4.6 permet de trouver dans $R$ des segments $\gamma_{1}^{\prime}$ et $\gamma_{2}^{\prime}$ de feuilles de $\mathcal{F}^{\prime}$ et de $\mathcal{G}^{\prime}$ se coupant transversalement en un point $x^{\prime}$. De plus, orientons ces segments de feuilles fuyant l'origine $(0,0)$. Notons $\gamma_{1}$ et $\gamma_{2}$ des segments de feuilles de $\mathcal{F}$ et $\mathcal{G}$ dans $R$ se coupant en un point $x$, et orientés en fuyant l'origine. La preuve du Lemme 4.6 montre que les segments $\gamma_{2}$ et $\gamma_{2}^{\prime}$ coupent simultanément de façon positive ou négative les segments $\gamma_{1}$ et $\gamma_{1}^{\prime}$, respectivement.

Comme $h$ préserve l'orientation, on en déduit que l'orientation en $h^{-1}\left(x^{\prime}\right)$ obtenue comme $\tilde{\mathcal{F}}$ suivie de $\tilde{\mathcal{G}}$, où ces feuilletages sont orientés en fuyant l'origine, est identique à celle obtenue en $x \operatorname{par} \mathcal{F}$ suivi de $\mathcal{G}$. Comme le signe de $\tilde{\beta}-\tilde{\alpha}$ est identique à celui de $\beta-\alpha$, on en déduit que $x^{\prime} \in \tilde{S}_{0}$. Comme $D_{\rho} \cap\left(S_{0} \backslash\left(C_{+}^{\eta} \cup C_{-}^{\eta}\right)\right)$ ne contient pas de point des courbes $D_{+}^{\prime}$ et $D_{-}^{\prime}$ et contient un point de $S_{0}^{\prime}$, il est inclus dans $S_{0}^{\prime}$, ce qui conclut la première inclusion annoncée.

Le même argument montre que, sur $D_{\rho} \backslash\left(S_{0} \cup\left(C_{+}^{\eta} \cup C_{-}^{\eta}\right)\right)$, les feuilletages $\mathcal{F}^{\prime}$ et $\mathcal{G}^{\prime}$ se coupent de façon topologiquement transverse et avec l'orientation inverse à celle dans $S_{0}^{\prime}$, ce qui conclut.

Pour achever la preuve de la Proposition 4.1 et donc du Théorème 1.1, il nous reste à montrer :

Proposition 4.3. Avec les hypothèses et notations ci-dessus, les nombres de translation relatifs $\rho(H, f)$ et $\rho\left(H^{\prime}, f^{\prime}\right)$ sont égaux.

Cette proposition représente le point le plus délicat de la preuve. En effet, dans la preuve de l'invariance du quotient $\alpha / \beta$, nous avons utilisé que les itérés arbitrairement grands $f^{\prime n}$ restent très près de $f^{n}$, car le feuilletage $\mathcal{F}$ guide $\mathcal{F}^{\prime}$ et donc les feuilles de $\mathcal{F}$ et 
de $\mathcal{F}^{\prime}$ définissant les applications de retour restent dans un même secteur arbitrairement fin. Par contre les applications $H$ et $H^{\prime}$ sont construites en suivant successivement des segments de feuilles des deux feuilletages $\mathcal{F}$ et $\mathcal{G}$, et $\mathcal{F}^{\prime}$ et $\mathcal{G}^{\prime}$. Pour $x$ et $x^{\prime}$ fixés proches, rien n'assure que $H^{n}(x)$ et $H^{\prime n}\left(x^{\prime}\right)$ seront comparables, si $n$ est choisi suffisamment grand. Nous allons ici présenter les idées permettant de résoudre cette difficulté, et de prouver la Proposition 4.3, en tentant d'éviter d'être trop technique. L'idée est que, quand $x$ est suffisamment proche de $0, H^{n}(x)$ et $H^{\prime n}\left(x^{\prime}\right)$ restent voisins pour $n$ de plus en plus grand. Il va donc falloir choisir d'abord l'itéré $n$ permettant d'approximer le nombre de translation $\rho(H ; f)$, et on choisira alors le voisinage de $(0,0)$ sur lequel on pourra en déduire une approximation de $\rho\left(H^{\prime} ; f^{\prime}\right)$.

Nous avons vu que les courbes $D_{+}^{\prime}$ et $D_{-}^{\prime}$ sont contenues, au voisinage de $(0,0)$ dans des cônes arbitrairement fins autour des droites $D_{+}$et $D_{-}$. De plus le Lemme 4.22 assure que le «cône» $S_{0}^{\prime}$, délimité par ces droites, et tel que les applications $H_{1}^{\prime}$ et $H_{2}^{\prime}$ sont les holonomies des restrictions $\mathcal{F}^{\prime}$ et $\mathcal{G}^{\prime}$ à $S_{0}^{\prime}$, correspond bien au cône $S_{0}$. Les segments de feuilles de $\mathcal{F}^{\prime}$ joignant un point $x^{\prime}$ à $H_{1}^{\prime}\left(x^{\prime}\right)$ seront incluses dans un $\mathcal{F}$-rectangle (de largeur arbitrairement petite si $x^{\prime}$ est pris proche de $(0,0)$ ) contenant un segment de feuille de $\mathcal{F}$ joignant un point $x$ à $H_{1}(x)$. Cette idée permet de montrer :

Lemme 4.23. Pour tout $\varepsilon>0$, il existe $\varepsilon_{1}>0$ et un voisinage $U_{\varepsilon}$ tel que :

(1) pour tout $x \in U_{\varepsilon} \cap D_{-}$et tout $x^{\prime} \in U_{\varepsilon} \cap D_{-}^{\prime}$ tels que $d\left(x, x^{\prime}\right)<\varepsilon_{1} \cdot\|x\|$ on a $d\left(H_{1}(x), H_{1}^{\prime}\left(x^{\prime}\right)\right)<\varepsilon \cdot\left\|H_{1}(x)\right\|$;

(2) pour tout $y \in U_{\varepsilon} \cap D_{+}$et tout $y^{\prime} \in U_{\varepsilon} \cap D_{+}^{\prime}$ tels que $d\left(y, y^{\prime}\right)<\varepsilon_{1} \cdot\|y\|$ on a $d\left(H_{2}^{-1}(y), H_{2}^{\prime-1}\left(y^{\prime}\right)\right)<\varepsilon \cdot\left\|H_{2}^{-1}(y)\right\|$;

(3) pour tout $x \in U_{\varepsilon} \cap D_{-}$et tout $x^{\prime} \in U_{\varepsilon} \cap D_{-}^{\prime}$ tels que $d\left(x, x^{\prime}\right)<\varepsilon_{1} \cdot\|x\|$, pour tout $n \in \mathbb{N}$ on a $d\left(f^{n}(x), f^{\prime n}\left(x^{\prime}\right)\right)<\varepsilon \cdot\left\|f^{n}(x)\right\|$.

Corollaire 4.4. Pour tout $\varepsilon>0$ et tout $n \in \mathbb{N}$, il existe $\delta>0$ et un voisinage $U_{\varepsilon}$ de $(0,0)$ tel que pour tout $x \in U_{\varepsilon} \cap D_{-}$et tout $x^{\prime} \in U_{\varepsilon} \cap D_{-}^{\prime}$ tels que $d\left(x, x^{\prime}\right)<\delta \cdot\|x\|$, on $\operatorname{ad}\left(H^{n}(x), H^{\prime n}\left(x^{\prime}\right)\right)<\varepsilon \cdot\left\|H^{n}(x)\right\|$.

De même que précédemment, nous allons utiliser un chapelet de perles pour contrôler la position relative, sur $D_{-}^{\prime}$, de points proches de points de $D_{-}$.

On fixe donc $\varepsilon_{0}>0$ et un chapelet (négatif) de $\varepsilon_{0}$-perles $\left(\Delta_{i}\right)$ couvrant l'une des demidroites de $D_{-}$, et invariant par les homothéties de rapport $\left.v \in\right] 0,1[$.

Lemme 4.24. Pour tout $r, s, t \in \mathbb{Z}, r>0, s>0$ tels que $t / r<\rho(H, f)<t / s$ il existe $l>0$ tel que, pour tout $x \in D_{-}$, les points $f^{r l}(x), f^{s l}(x)$ et $H^{t l}(x)$ appartiennent à des perles $\Delta_{i}, \Delta_{j}$ et $\Delta_{k}$, respectivement, avec $k-i \geq 5$ et $j-k \geq 5$.

Démonstration. La seule différence avec le Lemme 4.21 est que l'on demande ici que la propriété soit valable pour tout $x$ de la demi-droite. Ceci est une conséquence directe de l'invariance de $H$, de $f$ et du chapelet de perles par les homothéties de rapport $v \in] 0,1[$.

En utilisant le fait que la courbe $D_{-}^{\prime}$ est incluse, au voisinage de l'origine dans des secteurs radiaux arbitrairement fins autour de $D_{-}$, on montre facilement :

Lemme 4.25. Pour tout $\delta>0$ et tout voisinage $U$ de l'origine il existe $x \in U \cap D_{-}$et $x^{\prime} \in U \cap D_{-}^{\prime}$ tels que $d\left(x, x^{\prime}\right)<\delta \cdot\|x\|$. 
Le même raisonnement ayant montré comment déduire la Proposition $4.2 \mathrm{du}$ Lemme 4.19 montre que la Proposition 4.3 est conséquence directe du lemme suivant :

Lemme 4.26. Pour tout $r, s, t \in \mathbb{Z}, r>0, s>0$ tels que $t / r<\rho(H, f)<t / s$, il existe $l>0$ et $x^{\prime} \in D_{-}^{\prime}$ tel que $H^{\prime t l}\left(x^{\prime}\right)$ appartienne au segment $\left[f^{\prime r l}\left(x^{\prime}\right), f^{\prime s l}\left(x^{\prime}\right)\right]_{D_{-}^{\prime}}$.

Démonstration. On remarque qu'il existe $\varepsilon>0$ tel que, pour tout $x \in D_{-}$, la boule de centre $x$ et de rayon $\varepsilon\|x\|$ soit incluse dans une perle $\Delta_{i}$ : il suffit de voir que ceci est vrai, par compacité, sur tout segment de $D_{-} \backslash\{(0,0)\}$, puis d'utiliser l'invariance des perles par l'homothétie de rapport $v$.

On fixe alors l'entier $l>0$ donné par le Lemme 4.24.

On note alors $\delta>0$ et un voisinage $U_{\varepsilon}$ de l'origine, donnés par le Corollaire 4.4 pour $\varepsilon$ et $n=l t$. Quitte à restreindre $\delta$ et $U_{\varepsilon}$ si nécessaire, on peut supposer que l'item $3 \mathrm{du}$ Lemme 4.23 est aussi vérifié avec $\varepsilon_{1}=\delta$. On choisit alors, par le Lemme 4.25, deux points $x \in D_{-}, x^{\prime} \in D_{-}^{\prime}$ dans $U_{\varepsilon}$ tels que $d\left(x, x^{\prime}\right)<\delta\|x\|$.

On en déduit :

$$
\begin{gathered}
d\left(f^{l r}(x), f^{\prime l r}\left(x^{\prime}\right)\right)<\varepsilon\left\|f^{l r}(x)\right\| \\
d\left(f^{l s}(x), f^{\prime l s}\left(x^{\prime}\right)\right)<\varepsilon\left\|f^{l s}(x)\right\| \\
d\left(H^{l t}(x), H^{\prime l t}\left(x^{\prime}\right)\right)<\varepsilon\left\|H^{l t}(x)\right\| .
\end{gathered}
$$

Soient $i, j, k$, donnés par le Lemme 4.24, tels que $f^{r l}(x) \in \Delta_{i}, f^{s l}(x) \in \Delta_{j}$ et $H^{t l}(x) \in \Delta_{k}$. Soient $i^{\prime}, j^{\prime}, k^{\prime}$ tels que $f^{\prime l r}\left(x^{\prime}\right) \in \Delta_{i^{\prime}}, f^{\prime s l}\left(x^{\prime}\right) \in \Delta_{j^{\prime}}$ et $H^{\prime t l}\left(x^{\prime}\right) \in \Delta_{k^{\prime}}$. Alors $\left|i-i^{\prime}\right| \leq 1,\left|j-j^{\prime}\right| \leq 1$ et $\left|k-k^{\prime}\right| \leq 1$, ce qui implique $k^{\prime}-i^{\prime} \geq 3$ et $j^{\prime}-k^{\prime} \geq 3$, et donc le point $H^{\prime t l}\left(x^{\prime}\right)$ appartient au segment $\left[f^{\prime k r}\left(x^{\prime}\right), f^{\prime k s}\left(x^{\prime}\right)\right]_{D^{\prime}}$ ce qui conclut.

Remerciements. Nous remercions Robert Roussarie et Freddy Dumortier pour l'intérêt qu'ils ont porté à ce travail, ainsi que Fernando Sanz Sanchez qui nous a posé ce problème.

\section{A. Appendice : conclusion}

A.1. 'Pinch conjugacy' pour des difféomorphismes des surfaces. Soit $\gamma$ une connexion comme ci-dessus, et $\left(\mathcal{L}_{\alpha}, \mathcal{L}_{\beta}^{\lambda}\right)$ son couple caractéristique. Si $\alpha$ et $\beta$ sont de même signe, on construit aisément un rectangle $R$ plongé de sorte que $\gamma$ soit l'un des côtés de $R$, que $R \backslash \gamma$ soit transverse au champ de vecteurs, et que les deux côtés adjacents à $\gamma$ soient inclus dans les variétés invariantes de dimension deux $\left(W^{s}(p)\right.$ et $\left.W^{u}(q)\right)$. L'application de retour sur $R$ est alors un difféomorphisme $P_{X, R}$ de $R$, pour lequel $\gamma$ est une connexion entre les selles $p$ et $q$.

$\mathrm{Au}$ cours de discussions avec F. Dumortier, il nous est apparu que la notion d'équivalence topologique pour des champs de vecteurs $X, X^{\prime}$ au voisinage de telles connexions était très proche (peut-être équivalente) à la notion de pinch conjugacy (conjugaison pincée) des connexions des applications $P_{X, R}$ et $P_{X^{\prime}, R^{\prime}}$, étudiée dans [6]. De même que dans notre travail, [6] montrait que, suivant la transition, la connexion présentait, ou pas, des invariants pour la conjugaison pincée. Il serait intéressant d'interpréter leur condition sur la transition du difféomorphisme en terme du défaut de conformité de la transition du champ de vecteurs, puis d'exprimer leurs invariants en terme des invariants $(\alpha / \beta, \Psi(\alpha, \beta, t))$. 
A.2. Connexion incluse dans un cycle. On considère $\gamma$ une connexion comme cidessus entre des selles $p$ et $q$ et l'on suppose de plus que les variétés $W^{s}(p)$ et $W^{u}(q)$ (de dimension 2) se coupent transversalement le long d'une orbite $\sigma$ : autrement dit, les variétés invariantes de $p$ et $q$ forment un cycle.

Un tel cycle est génériquement accumulé par des orbites périodiques selles hyperboliques, appartenant à une même classe homocline. Nous pensons pouvoir montrer, dans un travail ultérieur (c.f. [5]), que le défaut de conformité $t$ de la transition de $\gamma$ ainsi que les invariants $(\alpha / \beta, \Psi(\alpha, \beta, t))$ sont reliés à l'existence de tangences homocliniques de ces orbites périodiques, dans des voisinages arbitrairement fins de $\gamma$.

\section{RÉFÉRENCES}

[1] G. R. Belitsky. Functional equations and conjugacy of local diffeomorphisms of a finite smoothness class. Funct. Anal. Appl. 7, (1973), 268-277.

[2] J. A. Beloqui. Modulus of stability for vector fields on 3-manifolds. J. Diff. Eq. 65 (1986), 374-395.

[3] C. Bonatti et S. Firmo. Feuilles compactes d'un feuilletage générique en codimension 1. Ann. Scient. Éc. Norm. Sup. 27 (1994), 407-462.

[4] M. J. Dias Carneiro et J. Palis. Bifurcations and global stability of families of gradients. Inst. Hautes Etudes Sci. Publ. Math. 70 (1990), 103-168.

[5] E. Dufraine. Équivalence topologique de champs de vecteurs en présence de connexions. Thèse, Université de Bourgogne, 2001.

[6] F. Dumortier et W. de Melo. A type of moduli for saddle connections of planar diffeomorphisms. J. Diff. Eq. 75(1) (1988), 88-102.

[7] J. Palis. A differentiable invariant of topological conjugacies and modulus of stability. Asterisque 51 (1978), 335-346.

[8] S. van Strien. One parameter families of vectorfields. Thesis, Rijksuniversiteit, 1982.

[9] F. Takens. Global phenomena in bifurcations of dynamical systems with simple recurrence. Jahresber. Deutsch. Math.-Verein. 81(2) (1978/79), 87-96.

[10] F. Takens. Moduli and bifurcations; nontransversal intersections of invariant manifolds of vectorfields. Functional Differential Equations and Bifurcation (Lecture Notes in Mathematics, 799). Springer, Berlin, 1980, pp. 368-384.

[11] G. Vegter. Bifurcations of gradient vector fields. Thesis, Groningen, 1983.

[12] G. Vegter. Global stability of generic two-parameter families of gradients on three-manifolds. Dynamical Systems and Bifurcations (Lecture Notes in Mathematics, 1125). Springer, 1985, pp. 107-129. 